\title{
TARGET PATTERNS IN A 2-D ARRAY OF OSCILLATORS WITH NONLOCAL COUPLING
}

\author{
GABRIELA JARAMILLO AND SHANKAR C. VENKATARAMANI
}

\begin{abstract}
We analyze the effect of adding a weak, localized, inhomogeneity to a two dimensional array of oscillators with nonlocal coupling. We propose and also justify a model for the phase dynamics in this system. Our model is a generalization of a viscous eikonal equation that is known to describe the phase modulation of traveling waves in reaction-diffusion systems. We show the existence of a branch of target pattern solutions that bifurcates from the spatially homogeneous state when $\varepsilon$, the strength of the inhomogeneity, is nonzero and we also show that these target patterns have an asymptotic wavenumber that is small beyond all orders in $\varepsilon$.

The strategy of our proof is to pose a good ansatz for an approximate form of the solution and use the implicit function theorem to prove the existence of a solution in its vicinity. The analysis presents two challenges. First, the linearization about the homogeneous state is a convolution operator of diffusive type and hence not invertible on the usual Sobolev spaces. Second, a regular perturbation expansion in $\varepsilon$ does not provide a good ansatz for applying the implicit function theorem since the nonlinearities play a major role in determining the relevant approximation, which also needs to be "correct" to all orders in $\varepsilon$. We overcome these two points by proving Fredholm properties for the linearization in appropriate Kondratiev spaces and using a refined ansatz for the approximate solution which was obtained using matched asymptotics.
\end{abstract}

\section{INTRODUCTION}

Reaction-Diffusion equations describe the evolution of quantities $u(x, t)$ that are governed by "local" nonlinear dynamics, given by a reaction term $F(u)$, coupled with Fickian diffusion,

$$
\frac{\partial u}{\partial t}=D \Delta u+F(u)
$$

They are generic models for patterns forming systems and have applications to a wide range of phenomena from population biology [Fis37, KRP37, chemical reactions Win73, KH81, Mer92, TF80a, fluid NW69, Seg69 and granular ER99, AT06 flow patterns, and in waves in neural [Fit61, NAY62, CH09] and in cardiac $\mathrm{GJP}^{+}$95] tissue.

The onset of self-organized patterns in reaction-diffusion models typically corresponds to a bifurcation where a steady equilibrium for the local dynamics $\dot{u}=F(u)$ loses stability either through a pitchfork bifurcation, giving a bistable medium, or through a Hopf bifurcation, giving an oscillatory medium. In the latter case, a defining feature is the occurrence of temporally periodic, spatially homogeneous states,

2010 Mathematics Subject Classification. primary: 47G20; secondary: 45M05, 35B36;

Key words and phrases. Target patterns, Nonlocal Eikonal equation, Kondratiev space, Fredholm operators, Asymptotics beyond all orders. 
and an analysis of the symmetries of the system show that they can generically give rise to traveling waves, spiral waves, and target patterns GSS88, DSSS05. A prototypical example of these behaviors is the Belousov-Zhabotinsky reaction where chemical oscillations are manifested as a change in the color of the solution. This system displays self-organized spiral waves, i.e. they form without any external forcing or perturbation, as well as target patterns, which in contrast form when an impurity is present at the center of the pattern [SM06, IPV87, TF80a].

As already mentioned, these patterns are not specific to oscillating chemical reactions and in fact can be seen in any spatially extended oscillatory medium. In this more general setting, and for the particular case of target patterns, an impurity or defect constitutes a localized region where the system is oscillating at a slightly different frequency from the rest of the medium. Depending on the sign of the frequency shift, and for systems of dimensions $d \leq 2$, these defects can act as pacemakers and generate waves that propagate away from the impurity. In the case when $d=2$, these waves are seen as concentric circular patterns that propagate away from the defect.

Showing the existence of these target pattern solutions in reaction-diffusion systems, and related amplitude equations, has been the subject of extensive research, see [KH81, TF80b, Hag81, Nag91, MM95, GKS00, SM06, KS07, Jar15, for some examples as well as the reference in $\mathrm{CH} 93$. Mathematically one can describe these patterns as modulated wave trains which correspond to solutions to (1) of the form $u(\mathbf{x}, t)=u_{*}(\phi(\mathbf{x}, t) ; \nabla \phi(\mathbf{x}, t))$, where $u_{*}(\xi ; \mathbf{k})$ is a $2 \pi$ periodic function of $\xi$ that depends on the wavenumber, $\mathbf{k}$ KH81, DSSS05, KS07. In other words, these patterns correspond to periodic traveling waves, whose phase varies slowly in time and space. Using multiple scale analysis one can show that the evolution of the phase, $\phi$, over long times is given by the viscous eikonal equation,

$$
\phi_{t}=\Delta \phi-|\nabla \phi|^{2} .
$$

This equation has been studied extensively in the physics literature, starting with the work of Kuramoto [KT76]. More recently it was shown that it does indeed provide a valid approximation for the phase modulation of the patterns seen in oscillatory media [DSSS05].

Since oscillating chemical reaction can be thought of as a continuum of diffusively coupled oscillators, it is not surprising that an analogue of the above equation can also be derived as a description for the phase dynamics for an array of oscillators. Indeed, in Appendix A we formally show that the following integro-differential equation provides a phase approximation for a slow-time, $\mathrm{O}(1)$ in space, description of nonlocally coupled oscillators,

$$
\phi_{t}=\mathcal{L} * \phi-|\mathcal{J} * \nabla \phi|^{2}+\varepsilon g(x, y) \quad(x, y) \in \mathbb{R}^{2} .
$$

This equation will be the focus of our paper. Here the operators $\mathcal{L}$ and $\mathcal{J}$ are spatial kernels that depend on the underlying nonlocal coupling between the oscillators. In particular, the convolution kernel $\mathcal{L}$ models the nonlocal coupling of these phase oscillators and can be thought of as an analog of $\Delta$. Similarly, the term $|\mathcal{J} * \nabla \phi|^{2}=\mathcal{J} * \nabla \phi \cdot \mathcal{J} * \nabla \phi$ represents nonlocal transport along diffused gradients and is a generalization of the quadratic nonlinearity of the viscous eikonal equation. Finally, the function $g(x, y)$ represents an inhomogeneity that perturbs the "local" frequency of the oscillators. Notice that the viscous eikonal equation 
is recovered when $\mathcal{L}$ and $\mathcal{J}$ are the Laplacian and the identity operator, respectively. This framework also incorporates other models for spatio-temporal pattern formation, including the Kuramoto-Sivashinsky equation which corresponds to $\mathcal{L}=-\Delta-\Delta^{2}, \mathcal{J}=$ Id .

Our mathematical motivation for studying the above model comes from its nonlocal aspect and the resulting analytical challenges. The approach we propose for studying the operator $\mathcal{L}$ is novel and could be adapted to study other problems which involve similar convolution operators. In particular, the type of linear operators that we will consider are a generalization of the kernels used in neural field models or continuum coupled models of granular flow. Just as in reaction-diffusion systems, these models exhibit spatio-temporal periodic patterns, bumps, and traveling waves, (see Coo05, Erm98, PE01, KB10a for the case of neural field models, and UMS98, VO98, VO01, AT06 for the case of patterns in granular flows). In particular, among the examples of traveling waves seen in experiments and replicated in the neural field models are spirals and target patterns $\mathrm{HTY}^{+} 04, \mathrm{~KB} 10 \mathrm{~b}, \mathrm{FB} 04$, which are of interest to us.

The challenge however is that, like our model (2), these systems are not amenable to methods from spatial dynamics which are typically used to study these phenomena. So we look for a more functional analytic approach, specifically using the implicit function theorem, for proving the existence of solutions. As with other integro-differential equations the difficulty comes from the linearization, which is a noncompact convolution operator, and in general not invertible when considered as a map between Sobolev spaces. This is a significant analytical challenge, and one way to overcome this difficulty is to use specific convolution kernels that allow for these integro-differential equations to be converted into PDEs via the Fourier Transform [LT03, or in the radially symmetric case use sums of modified Bessel functions as models for synaptic footprint to simplify the analysis [FB04].

The approach we consider in this paper is broader as it allows us to consider a larger class of convolution kernels by showing that these operators are Fredholm in appropriate weighted spaces. This approach is similar to the one in JS16, where we treated the one dimensional case and showed existence of target patterns in a large one dimensional array of oscillators with nonlocal coupling. For the applications we have in mind, e.g. neural field models, we need to extend these results to two dimensional arrays.

The two dimensional case is technically more interesting because a regular perturbation expansion in $\varepsilon$ does not always provide the correct ansatz. Indeed, in the case with local coupling, the equation

$$
-\omega=\Delta \tilde{\phi}-|\nabla \tilde{\phi}|^{2}+\varepsilon g(x, y), \quad(x, y) \in \mathbb{R}^{2}
$$

which results from inserting the ansatz $\phi(x, y, t)=\tilde{\phi}(x, y)-\omega t$ into the perturbed viscous eikonal equation, is conjugate to a Schrödinger eigenvalue problem via the Hopf-Cole transform, $\tilde{\phi}=-\ln (\Psi)$ :

$$
\omega \Psi=\Delta \Psi-\varepsilon g(x, y) \Psi, \quad(x, y) \in \mathbb{R}^{2} .
$$

In two dimensions, it is well known that the Schrödinger eigenvalue problem has bound states if $\varepsilon \int g<0$ Sim76]. Notice that the ground state eigenfunction $\Psi_{0}$ can be chosen to be everywhere positive, so that $-\ln \left(\Psi_{0}\right)-\omega t$ does define a phase function $\phi$ solving the viscous eikonal equation with inhomogeneity. At the same time, the eigenvalue corresponding to the ground state is small beyond all orders 
of $\varepsilon$ (see Sim76 and Section 2 below), and is therefore not accessible to a regular perturbation expansion. This is the other analytical challenge that we have to overcome, and our approach is to develop a superasymptotic perturbation expansion for $\phi$, i.e. an approximation whose error is $\mathrm{O}(\exp (-|c / \varepsilon|))$ and captures behaviors that are small beyond all orders in $\varepsilon$, Boy99.

To show the existence of traveling waves for equation (2) we make the following assumptions on the convolution kernels $\mathcal{L}$ and $J$. First, we assume the kernel $\mathcal{L}$ is a diffusive and exponentially localized kernel that commutes with rotations. Consequently, its Fourier symbol $L$ depends only on $\xi=|\mathbf{k}|^{2}$. We also impose additional properties that we specify in the Hypotheses 1.1 and 1.2 . A representative example to keep in mind throughout the paper is the convolution kernel that would result in the formal operator $\Delta(\operatorname{Id}-\Delta)^{-1}$.

We reiterate that the model (2) is derived under the assumption that the phase $\phi(x, t)$ varies slowly in time, with no assumptions on its spatial variation. If we assume that the solutions also vary slowly in space, then hypothesis 1.2 implies that the nonlocal operator $\mathcal{L}$ can be (formally) replaced by $\Delta$, and 2 reduces to the "local" viscous eikonal equation. Indeed, this is the setting for a substantial body of work on weakly coupled nonlinear oscillators Kur84, SL12. Our additional contribution is that we rigorously show the existence of target solutions of (2) that vary slowly (on a scale $\sim e^{1 / \epsilon}$ ) in space and time, if the model satisfies:

Hypothesis 1.1. The multiplication operator $L$ is a function of $\xi:=|\mathbf{k}|^{2}$. Its domain can be extended to a strip in the complex plane, $\Omega=\mathbb{R} \times\left(-\mathrm{i} \xi_{0}, \mathrm{i} \xi_{0}\right)$ for some sufficiently small and positive $\xi_{0} \in \mathbb{R}$, and on this domain the operator is uniformly bounded and analytic. Moreover, there is a constant $\xi_{m} \in \mathbb{R}$ such that the operator $L(\xi)$ is invertible with uniform bounds for $|\operatorname{Re} \xi|>\xi_{m}$.

Our main result, Theorem 1 requires $\ell=1$ in the following hypothesis. We state the hypothesis in more generality because some of the intermediate results also hold more generally with $\ell \geq 1$.

Hypothesis 1.2. The multiplication operator $L(\xi)$ has a zero, $\xi^{*}$, of multiplicity $\ell \geq 1$ which we assume is at the origin. Therefore, the symbol $L(\xi)$ admits the following Taylor expansion near the origin.

$$
L(\xi)=(-\xi)^{\ell}+\mathrm{O}\left(\xi^{\ell+1}\right), \quad \text { for } \quad \xi \sim 0 .
$$

Hypothesis 1.3. The kernel $\mathcal{J}$ is radially symmetric, exponentially localized, twice continuously differentiable, and

$$
\int_{\mathbb{R}^{2}} \mathcal{J}(\mathbf{x}) d \mathbf{x}=1
$$

Our strategy to show the existence of traveling waves will be to first establish the Fredholm properties of the convolution operator $\mathcal{L}$ following the ideas described in [JSW17]. This will allow us to precondition our equation by an operator $\mathscr{M}$, resulting in an equation which has as its linear part the Laplace operator. We then proceed to show the existence of target patterns in the nonlocal problem. More precisely, we prove Theorem 1 where we use the following notation:

- Here $L_{\sigma}^{2}\left(\mathbb{R}^{2}\right)$ denotes the $L^{2}$ space with weight $\left(1+|\mathbf{x}|^{2}\right)^{\sigma / 2}$.

- Similarly, $H_{\sigma}^{s}\left(\mathbb{R}^{2}\right)$ denotes the Hilbert space $H^{s}$ with weight $\left(1+|\mathbf{x}|^{2}\right)^{\sigma / 2}$. 
- Lastly, the symbol $M_{\sigma}^{s, p}\left(\mathbb{R}^{2}\right)$ describes the completion of $C_{0}\left(\mathbb{R}^{2}\right)$ functions under the norm

$$
\|u\|_{M_{\sigma}^{s, p}\left(\mathbb{R}^{d}\right)}^{p}=\sum_{|\alpha| \leq s}\left\|D^{\alpha} u(\mathbf{x}) \cdot\left(1+|\mathbf{x}|^{2}\right)^{(\sigma+|\alpha|) / 2}\right\|_{L^{p}\left(\mathbb{R}^{d}\right)}^{p} .
$$

We describe these last spaces with more detail in Section 3 .

Theorem 1. Suppose that the kernels $\mathcal{L}$ and $\mathcal{J}$ satisfy Hypotheses 1.1. 1.2 with $\ell=1$, and 1.3. Additionally, suppose $g$ is in the space $L_{\sigma}^{2}\left(\mathbb{R}^{2}\right)$ with $\sigma>1$ and let $M=\frac{1}{2 \pi} \int_{\mathbb{R}^{2}} g<0$. Then, there exists a number $\varepsilon_{0}>0$ and a $C^{1}$ map

$$
\begin{array}{ccc}
\Gamma:\left[0, \varepsilon_{0}\right) & \longrightarrow & \mathcal{D} \subset M_{\gamma-1}^{2,1}\left(\mathbb{R}^{2}\right) \\
\varepsilon & \longmapsto & \psi
\end{array}
$$

where $\gamma>0$ and $\mathcal{D}=\left\{\phi \in M_{\gamma-1}^{1,2}\left(\mathbb{R}^{2}\right) \mid \nabla \phi \in H_{\gamma}^{1}\left(\mathbb{R}^{2}\right)\right\}$, that allows us to construct an $\varepsilon$-dependent family of target pattern solutions to (2). Moreover, these solutions have the form

$$
\Phi(r, \theta, t ; \varepsilon)=-\chi(\lambda(\varepsilon) r) \ln \left(K_{0}(\lambda(\varepsilon) r)\right)+\psi(r, \theta ; \varepsilon)-\lambda^{2}(\varepsilon) t, \quad \lambda(\varepsilon)>0 .
$$

In particular, as $r \rightarrow \infty$,

- $\psi(r ; \varepsilon)<C r^{-\delta}$, for $C \in \mathbb{R}$ and $\delta \in(0,1)$; and

- $\lambda(\varepsilon)^{2} \sim 4 C(\varepsilon) \mathrm{e}^{-2 \gamma_{e}} \exp \left(\frac{2}{\varepsilon M}\right)$, where $C(\varepsilon)$ represents a constant that depends on $\varepsilon$, and $\gamma_{e}$ is the Euler constant.

In addition, these target pattern solutions have the following asymptotic expansion for their wavenumber

$$
k(\varepsilon) \sim \exp \left(\frac{1}{\varepsilon M}\right)+\mathrm{O}\left(\frac{1}{r^{\delta+1}}\right) \quad \text { as } \quad r \rightarrow \infty .
$$

Remark 1.4. If $g \in L_{\sigma}^{2}\left(\mathbb{R}^{2}\right)$ with $\sigma>1$, it follows that

$$
\begin{aligned}
& \int_{\mathbb{R}^{2}}|g|\left(1+|\mathbf{x}|^{2}\right)^{(\sigma-1) / 3} d \mathbf{x} \leq\left[\int_{\mathbb{R}^{2}}|g|^{2}\left(1+|\mathbf{x}|^{2}\right)^{\sigma} d \mathbf{x} \int_{\mathbb{R}^{2}}\left(1+|\mathbf{x}|^{2}\right)^{-1-(\sigma-1) / 3} d \mathbf{x}\right]^{1 / 2}<\infty, \\
& \text { and } \\
& \int_{\mathbb{R}^{2}}|g|^{2 \sigma /(\sigma+1)} d \mathbf{x} \leq\left[\int_{\mathbb{R}^{2}}|g|^{2}\left(1+|\mathbf{x}|^{2}\right)^{\sigma} d \mathbf{x}\right]^{\sigma /(\sigma+1)}\left[\int_{\mathbb{R}^{2}}\left(1+|\mathbf{x}|^{2}\right)^{-\sigma^{2}} d \mathbf{x}\right]^{1 /(\sigma+1)}<\infty .
\end{aligned}
$$

Consequently, there is $\delta>0$ such that $\int_{\mathbb{R}^{2}}|g(\mathbf{x})|\left(1+|\mathbf{x}|^{\delta}\right) d \mathbf{x}<\infty$ and $\int_{\mathbb{R}^{2}}|g(\mathbf{x})|^{1+\delta} d \mathbf{x}<$ $\infty$, so that the Schrödinger operator $-\Delta+\varepsilon g$ satisfies the hypothesis in [Sim76].

Remark. Here and henceforth in the paper the function $\chi(x) \in C^{\infty}(\mathbb{R})$ is a cut off function, whose precise form is immaterial, and satisfies $\chi(x)=1$ for $|x|>2$ and $\chi(x)=0$ for $|x|<1$.

The rest of this paper is organized as follows: In Section 2 we analyze the case with local coupling and show the existence of traveling waves for the viscous eikonal equation using matched asymptotics. In Section 3 we review properties of Kondratiev spaces and state Fredholm properties of the Laplacian and related operators, leaving the proofs of these results for the appendices. Finally, in Section 4 we derive Fredholm properties for the convolution operator $\mathcal{L}$ and then, guided by the results from Section 2, we proceed to prove Theorem 11. We present a formal derivation of the nonlocal eikonal equation (2) in Appendix A In Appendix B, we prove various subsidiary results that are needed for the proof of Theorem 1 


\section{Matched ASymptotics For 2D TARget PAtterns}

As we discussed in the introduction, the viscous eikonal equation

$$
\partial_{t} \phi=\Delta \phi-|\nabla \phi|^{2}+\varepsilon g(x, y), \quad(x, y) \in \mathbb{R}^{2} .
$$

is an abstract model for the evolution of the phase of an array of oscillators with nearest neighbor coupling DSSS05. The perturbation $\varepsilon g(x, y)$, a localized function, represents a small patch of oscillators with a different frequency than the rest of the network. It is well known that this system can produce target patterns that bifurcate from the steady state, $\phi=0$, when the parameter $\varepsilon$ is of the appropriate sign. Our aim in this section is to determine an accurate approximation to these target wave solutions using a formal approach based on matched asymptotics.

We therefore consider solutions to equation (3) of the form $\phi(x, y, t)=\tilde{\phi}(x, y)-\omega t$, where $\tilde{\phi}$ solves

$$
-\omega=\Delta \tilde{\phi}-|\nabla \tilde{\phi}|^{2}+\varepsilon g(x, y), \quad(x, y) \in \mathbb{R}^{2}, \quad \omega>0 .
$$

We also assume in this section that $g$ is a radial and algebraically localized function that satisfies

$$
\int_{0}^{\infty}|g(r)|(1+r)^{\sigma} r d r<\infty, \quad \text { for some } \quad \sigma>0 .
$$

This simplifies our analysis since we can restrict ourselves to finding radially symmetric solutions. In addition, because the viscous eikonal equation (4) only depends on derivatives of $\tilde{\phi}$, we can recast it as a first order ODE for the wavenumber $\zeta=\tilde{\phi}_{r}$ :

$$
\zeta_{r}+\frac{\zeta}{r}-\zeta^{2}+\varepsilon g(r)=-\omega
$$

Here, $\omega=\omega(\varepsilon)$ is an eigenparameter, i.e. it is not specified, rather it is determined in such a way as to ensure that the solutions satisfy the required boundary conditions.

For radial solutions that are regular at the origin, $\zeta(r)$ is $O(r)$ as $r \rightarrow 0$, and we can rewrite the ODE in an equivalent integral form

$$
\zeta(r)=\frac{1}{r} \int_{0}^{r}\left[\zeta^{2}(\eta)-\omega-\varepsilon g(\eta)\right] \eta d \eta .
$$

Moreover, because we are bifurcating from the trivial state $\zeta=0$, we can assume that $\zeta$ is small if $\varepsilon$ is small, and posit the following regular expansions

$$
\begin{aligned}
\zeta & =\varepsilon \zeta_{1}+\varepsilon^{2} \zeta_{2}+\varepsilon^{3} \zeta_{3}+\cdots, \\
\omega & =\varepsilon \omega_{1}+\varepsilon^{2} \omega^{2}+\varepsilon^{3} \omega_{3}+\cdots .
\end{aligned}
$$

Substituting these expressions in (7), we obtain that at $O(\varepsilon)$,

$$
\zeta_{1}=-\frac{\omega_{1} r}{2}-\frac{1}{r} \int_{0}^{r} g(\eta) \eta d \eta
$$

Now, because we are interested in target patterns, solutions should satisfy $\zeta \rightarrow$ $k(\varepsilon)>0$ as $r \rightarrow \infty$, where $k(\varepsilon)$ is the asymptotic wavenumber. This requires us to consider functions $\zeta_{1}$ that have a finite limit as $r \rightarrow \infty$, and forces us to pick $\omega_{1}=0$. The result is that $r \zeta_{1}(r) \rightarrow-\int g(r) r d r=-M<\infty$, in the limit of $r$ going to infinity.

At the same time, from assumption (5) on the algebraic localization of $g$, we have the quantitative estimate

$$
\left|M-r \zeta_{1}(r)\right| \leq \frac{1}{(1+r)^{\sigma}} \int_{r}^{\infty}|g(\eta)|(1+\eta)^{\sigma} \eta d \eta \leq C(1+r)^{-\sigma}
$$


so that at order $O\left(\varepsilon^{2}\right)$ we find

$$
\zeta_{2}=-\frac{\omega_{2} r}{2}+\frac{1}{r} \int_{0}^{r} \zeta_{1}^{2} \eta d \eta
$$

with $\zeta_{1}^{2}$ in $L^{1}\left(\mathbb{R}^{2}\right)$. Again the boundary conditions force $\omega_{2}=0$, and as a result we obtain $\zeta_{2} \sim \frac{M^{2}}{r} \ln \left(\frac{r}{r_{c}}\right)$ for large values of $r$. Here the constant $r_{c}$ depends on $g$ and is given by the following limit

$$
\ln \left(r_{c}\right)=\lim _{r \rightarrow \infty}\left[\ln (r)-\frac{1}{M^{2}} \int_{0}^{r} \zeta_{1}^{2} \eta d \eta\right],
$$

which we know exists from the estimate for $\left|M-r \zeta_{1}(r)\right|$.

Note that these expressions for $\omega_{2}$ and $\zeta_{2}$ imply that the asymptotic wavenumber is $o\left(\varepsilon^{2}\right)$. In fact, continuing this procedure it is easy to check that we get $\omega_{j}=0$ for all $j$, so that the frequency $\omega$ is o $\left(\varepsilon^{n}\right)$ for all orders in $\varepsilon$. In addition, the expressions for $\zeta_{1}$ and $\zeta_{2}$ yield the expansion

$$
\zeta \approx-\frac{\varepsilon M}{r}+\frac{\varepsilon^{2} M^{2} \ln \left(r / r_{c}\right)}{r}+\cdots .
$$

whose terms are not uniformly ordered. For instance,

$$
\left|\varepsilon^{2} \zeta_{2}\right| \geq\left|\varepsilon \zeta_{1}\right| \quad \text { for } \quad r \geq r_{c} \exp \left(\frac{1}{\varepsilon|M|}\right) .
$$

This suggests that the above inner expansion is not uniformly valid. We therefore need to introduce an outer expansion and match both solution in an intermediate region given by $r \sim r_{c} \exp \left(\left|\varepsilon^{-1} M^{-1}\right|\right)$.

In this intermediate region the inhomogeneity, $\varepsilon g(r)$, and the frequency, $\omega$, are small compared to the other terms in the equation, so that the radial eikonal equation (6) reduces to

$$
\zeta_{r}+\frac{\zeta}{r}-\zeta^{2} \approx 0
$$

We can solve this explicitly to find that

$$
\zeta=\frac{1}{r(C-\ln (r))},
$$

where $C=C(\varepsilon)$ is a, possibly $\varepsilon$ dependent, constant of integration. Comparing this result with the inner expansion $(8)$ leads to $C \approx \frac{-1}{\varepsilon M}$, to leading order. We can then write $C=-(\varepsilon M)^{-1}+c_{0}+c_{1} \varepsilon+\ldots$, and for fixed $r$ and as $\varepsilon \rightarrow 0$, obtain

$$
\zeta=\frac{\varepsilon M}{r}+\frac{\varepsilon^{2} M^{2}\left(\ln (r)-c_{0}\right)}{r}+\cdots .
$$

Comparing again with the inner expansion (8), we see that $r_{c}=\exp \left(c_{0}\right)$.

In the outer region, where we retain the frequency, $\omega$, and neglect the inhomogeneity, g, solutions are described by the equation

$$
\zeta_{r}+\frac{\zeta}{r}-\zeta^{2}=-\omega
$$

If we define the 'outer' variable $\xi=\sqrt{\omega} r$ and scaling function $F$ so that $\zeta(r)=$ $\sqrt{\omega} F(\sqrt{\omega} r)$, then $F$ satisfies the $\omega$ (and hence also $\varepsilon$ ) independent equation $F_{\xi}+$ 
TABLE 1. In this section we will make use of the asymptotic behavior of $K_{0}(z)$ and its derivative $\partial_{z} K_{0}(z)=-K_{1}(z)$ (see Ref. AS92), which we summarize in this table

\begin{tabular}{ccc}
\hline & $z \rightarrow 0$ & $z \rightarrow \infty$ \\
\hline$K_{0}(z)$ & $-\ln (z / 2)-\gamma_{e}+\mathrm{O}\left(z^{2}|\ln z|\right)$ & $\sqrt{\frac{\pi}{2 z}} e^{-z}(1+\mathrm{o}(1 / z))$ \\
$K_{1}(z)$ & $\frac{1}{z}+\mathrm{O}(z|\ln z|)$ & $\sqrt{\frac{\pi}{2 z}} e^{-z}(1+\mathrm{o}(1 / z))$ \\
\hline
\end{tabular}

$F / \xi-F^{2}=-1$. Using the (differentiated) Hopf-Cole transformation $F(\xi)=-\psi^{\prime}(\xi) / \psi(\xi)$, and then solving for $\psi$ gives

$$
F(\xi)=-\frac{\partial_{\xi} K_{0}(\xi)}{K_{0}(\xi)}
$$

where $K_{0}(\xi)$ is the modified Bessel's function of the first kind WW96. Consequently, for $\xi=\sqrt{\omega} r \ll 1$ fixed and $\varepsilon \rightarrow 0$, a solution $\zeta$ of the outer equation is given by

$$
\zeta(r) \sim \frac{1}{r\left(-\ln (\sqrt{\omega r} / 2)-\gamma_{e}\right)},
$$

where $\gamma_{e}=0.5772 \ldots$ is the Euler constant AS92. This approximation is also valid in the intermediate region, allowing us to match it to the inner expansion,

$$
\frac{1}{r\left(-\frac{1}{\varepsilon M}+\ln \left(r_{c}\right)-\ln (r)\right)} \approx \frac{1}{r\left(-\frac{1}{2} \ln \omega+\ln 2-\gamma_{e}-\ln (r)\right)},
$$

and obtain the following approximation for the frequency

$$
\omega \sim \frac{4 \exp \left(-2 \gamma_{e}\right)}{r_{c}^{2}} \exp \left(\frac{2}{\varepsilon M}\right) .
$$

Hence, if we assume $\varepsilon M<0$, this does indeed show that $\omega$ is small beyond all orders in $\varepsilon$.

Remark. The viscous eikonal equation (3) is conjugate to a Schrödinger eigenvalue problem via the Hopf-Cole transform. The frequency $\omega=-\partial_{t} \phi$ in the viscous eikonal equation corresponds to the ground state energy for the Schrödinger operator $\Delta-$ $\varepsilon g(r)$. Indeed, the expression above is a refinement of the results from [Sim76] and KS07. for the ground state eigenvalue/frequency respectively, in that we have an expression for the numerical prefactor.

Remark. Integrating $\zeta=\partial_{r} \phi$, we can determine the phase $\phi(r)$ for the target patterns. From (8) and (9) we get the inner and outer expansions for the phase $\phi$

$$
\phi(r) \sim \phi_{0}+ \begin{cases}-\ln \left[\frac{-1}{\varepsilon M}+c_{0}+c_{1} \varepsilon+\cdots-\ln (r)\right] & r \text { fixed, } \varepsilon \rightarrow 0 \\ -\ln \left(K_{0}(\sqrt{\omega} r)\right) & \xi=\sqrt{\omega} r \text { fixed }, \varepsilon \rightarrow 0\end{cases}
$$

where $\phi_{0}$ is an arbitrary phase shift corresponding to a constant of integration.

The matching procedure above relied strongly on the coupling kernels $\mathcal{L}$ and $J$ being local, and having a radial inhomogeneity $g(r)$, so the results do not immediately carry over to the case of nonlocal coupling and/or nonradial and algebraically 


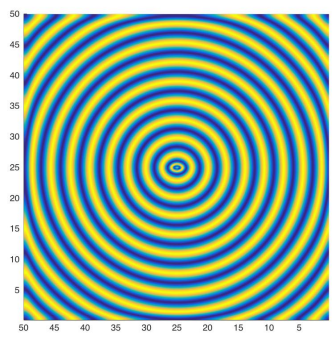

(a)

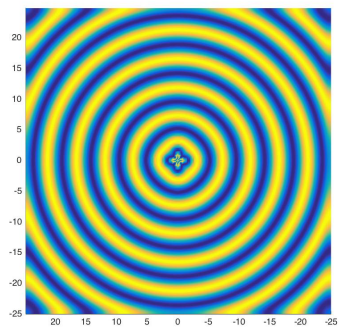

(c)

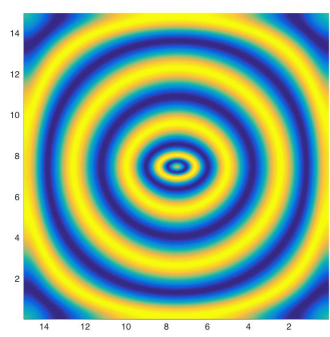

(b)

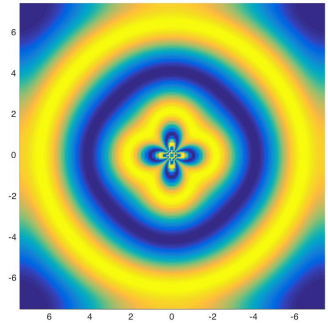

(d)

Figure 1. $g(x, y)=\frac{10}{\left(1+3 x^{2}+y^{2}\right)^{3 / 2}}$ in Figures (a) and (b). Notice how the pattern in the far field is radially symmetric, Figure (a), where as near the inhomogeneity it is elliptical, Figure (b). On the other hand, Figures (c) and (d) correspond to a system perturbed by $g(r, \theta)=\frac{10(1+\cos (4 \theta))}{(1+r)^{3}}$. Again notice how the pattern is radially symmetric in the far field, Figure (c), even though the inhomogeneity, $g$, is not radially symmetric, which results in a nonradially symmetric core, Figure (d).

localized inhomogeneities. Figure1 depicts numerical results for particular cases of the nonlocal eikonal equation (2) given by

$$
\partial_{t} \phi=(\operatorname{Id}-\Delta)^{-1} \Delta \phi-\left|\nabla(\operatorname{Id}-\Delta)^{-1} \phi\right|^{2}+\varepsilon g(x, y), \quad(x, y) \in \mathbb{R}^{2},
$$

with $\varepsilon=-0.5$ and $g(x, y)=10 *\left(1+3 x^{2}+y^{2}\right)^{-3 / 2}$ in 1(a) and (b) (respectively $g(r, \theta)=10 *(1+\cos (4 \theta)) /(1+r)^{3}$ in 1 (c) and $\left.(\mathrm{d})\right)$. This evolution equation was integrated using a spectral discretization for the spatial operator and exponential time differencing (ETD) for the time stepping CM02, KT05. The simulations where done for a box of length $L=100$ with $N=512$ grid points and a time step of $h=0.5$, see the code at Jar18. We will present a full discussion of our numerical methods and results in future work; here we only note that the far-field behavior of the target waves are (nearly) radially symmetric (see Figure 1) even in the general nonlocal problem. Indeed, setting $\partial_{t} \phi=-\omega$ with $\omega>0$, and rescaling to the "outer variables" $\tilde{x}=\sqrt{\omega} x, \tilde{y}=\sqrt{\omega} y$, we get $\tilde{\nabla}=\omega^{-1 / 2} \nabla, \tilde{\Delta}=\omega^{-1} \Delta$. Further, from the 
algebraic localization of $g$, it follows that

$$
\frac{1}{\omega} g\left(\omega^{-1 / 2} \tilde{x}, \omega^{-1 / 2} \tilde{y}\right) \sim \mathrm{O}\left(\omega^{\sigma}\right) \rightarrow 0 \text { as } \omega \rightarrow 0 .
$$

Consequently, the (formal) $\varepsilon \rightarrow 0$ limit equation in the outer variables is the viscous eikonal equation

$$
-1=(\operatorname{Id}-\omega \tilde{\Delta})^{-1} \tilde{\Delta} \phi-\left|\tilde{\nabla}(\mathrm{Id}-\omega \tilde{\Delta})^{-1} \phi\right|^{2}+\varepsilon \omega^{-1} g\left(\omega^{-1 / 2} \tilde{x}, \omega^{-1 / 2} \tilde{y}\right) \approx \tilde{\Delta} \phi-|\tilde{\nabla} \phi|^{2} .
$$

This argument, together with the numerical results depicted in Figure 1, suggests that, even for general inhomogeneities, we may approximate the dynamics of target waves solutions in the outer region by a radial viscous eikonal equation

$$
-\omega=\partial_{r r} \phi+\frac{1}{r} \partial_{r} \phi-\left(\partial_{r} \phi\right)^{2}
$$

This intuition will guide our analysis of the nonlocal equation (2) for general coupling kernels and nonradially symmetric, algebraically localized perturbations $g$. We will show that the frequency indeed scales as $\omega \sim \exp \left(\frac{2}{\varepsilon M}\right)$, with $\varepsilon M<0$, and that the target waves that bifurcate from the steady state are radially symmetric far away from the inhomogeneity. Our strategy consists of first finding, in Section 4.2. solutions to equation (2), with $\omega=0$, which give the appropriate intermediate approximation. Then in Section 4.3 we find the "outer" solutions to equation (2) by treating the frequency $\omega$ as an extra parameter. Finally, in Section 4.4 we derive a relation between the frequency $\omega$ and the parameter $\varepsilon$ using asymptotic matching, and then proceed to prove the results of Theorem 1 .

\section{Weighted Spaces}

We define the Kondratiev space [McO79], $M_{\gamma}^{s, p}\left(\mathbb{R}^{d}\right)$, with $d \in \mathbb{N}, s \in \mathbb{N} \cup\{0\}, \gamma \in$ $\mathbb{R}, p \in(1, \infty)$, as the space of locally summable, $s$ times weakly differentiable functions $u: \mathbb{R}^{d} \rightarrow \mathbb{R}$ endowed with the norm

$$
\|u\|_{M_{\gamma}^{s, p}\left(\mathbb{R}^{d}\right)}^{p}=\sum_{|\alpha| \leq s}\left\|D^{\alpha} u(\mathbf{x}) \cdot\left(1+|\mathbf{x}|^{2}\right)^{(\gamma+|\alpha|) / 2}\right\|_{L^{p}\left(\mathbb{R}^{d}\right)}^{p} .
$$

From the definition, it is clear that these spaces admit functions with algebraic decay or growth, depending on the weight $\gamma$, and that these functions gain localization with each derivative. Moreover, given real numbers $\alpha, \beta$, such that $\alpha>\beta$, the embedding $M_{\alpha}^{s, p}\left(\mathbb{R}^{d}\right) \subset M_{\beta}^{s, p}\left(\mathbb{R}^{d}\right)$ holds, and additionally if $s$ and $r$ are integers such that $s<r$ then $M_{\gamma}^{s, p}\left(\mathbb{R}^{d}\right) \subset M_{\gamma}^{r, p}\left(\mathbb{R}^{d}\right)$. As in the case of Sobolev spaces, we may identify the dual $\left(M_{\gamma}^{s, p}\left(\mathbb{R}^{d}\right)\right)^{*}$ with the space $M_{-\gamma}^{-s, q}\left(\mathbb{R}^{d}\right)$, where $p$ and $q$ are conjugate exponents, and in the case when $p=2$ we also have that Kondratiev spaces are Hilbert spaces. In particular, given $f, g \in M_{\gamma}^{s, 2}\left(\mathbb{R}^{d}\right)$ the pairing

$$
\langle f, g\rangle:=\sum_{|\alpha| \leq s} \int_{\mathbb{R}^{d}} D^{\alpha} f(\mathbf{x}) \cdot D^{\alpha} g(\mathbf{x}) \cdot\left(1+|\mathbf{x}|^{2}\right)^{(\gamma+|\alpha|)} d x
$$

satisfies all the properties of an inner product. This is not hard to see once we notice that for every $f \in L_{\gamma}^{2}\left(\mathbb{R}^{d}\right)$ the function $\left.f(\mathbf{x}) \dot{(1}+|\mathbf{x}|^{2}\right)^{\gamma / 2}$ is in the familiar Hilbert space $L^{2}\left(\mathbb{R}^{d}\right)$.

We will use this last property to decompose the space $M_{\gamma}^{s, 2}\left(\mathbb{R}^{d}\right)$ into a direct sum of its polar modes. Here we restrict ourselves to the case $d=2$ which is relevant for our analysis, but mention that a similar decomposition is possible in higher 
dimensions. More precisely, using the notation $M_{r, \gamma}^{s, p}\left(\mathbb{R}^{d}\right)$ to denote the subspace of radially symmetric functions in $M_{\gamma}^{s, p}\left(\mathbb{R}^{d}\right)$, we show that

Lemma 3.1. Given $s \in \mathbb{N} \cup\{0\}$ and $\gamma \in \mathbb{R}$, the space $M_{\gamma}^{s, 2}\left(\mathbb{R}^{2}\right)$ can be written as a direct sum decomposition

$$
M_{\gamma}^{s, 2}\left(\mathbb{R}^{2}\right)=\bigoplus m_{\gamma}^{n}
$$

where $n \in \mathbb{Z}$ and

$$
m_{\gamma}^{n}=\left\{u \in M_{\gamma}^{s, 2}\left(\mathbb{R}^{2}\right) \mid u=w(r) \mathrm{e}^{\mathrm{i} n \theta} \text { and } w(r) \in M_{r, \gamma}^{s, 2}\left(\mathbb{R}^{2}\right)\right\} .
$$

The proof of this result follows the analysis of Stein and Weiss in [SW16].

Proof. We need to show that each element $f \in M_{\gamma}^{s, p}\left(\mathbb{R}^{2}\right)$ can be well approximated by an element in the direct sum $\oplus m_{\gamma}^{n}$. To obtain a candidate function in the latter space we first identify $\mathbb{R}^{2}$ with the complex plane $\mathbb{C}$ by letting $z=x+\mathrm{i} y$ for $(x, y) \in \mathbb{R}^{2}$. Then, using the notation $z=r \mathrm{e}^{\mathrm{i} \theta}$ we write $f(z)=f\left(r \mathrm{e}^{\mathrm{i} \theta}\right)$ for each function $f \in M_{\gamma}^{s, 2}\left(\mathbb{R}^{2}\right)$. By Fubini's Theorem the function $f\left(r \mathrm{e}^{\mathrm{i} \theta}\right)$ is in $H^{s}([0,2 \pi])$ for a.e. $r \in[0, \infty)$, so we may express this function as a Fourier series in $\theta$.

$$
f\left(r \mathrm{e}^{\mathrm{i} \theta}\right)=\sum_{n \in \mathbb{Z}} f_{n}(r) \mathrm{e}^{\mathrm{i} n \theta}, \quad \text { where } \quad f_{n}(r)=\frac{1}{2 \pi} \int_{0}^{2 \pi} f\left(r \mathrm{e}^{\mathrm{i} \theta}\right) \cdot \mathrm{e}^{-\mathrm{i} n \theta} d \theta
$$

Notice that the functions $f_{n}(r)$ are in the space $M_{r, \gamma}^{s, 2}\left(\mathbb{R}^{2}\right)$, so that this sum is the desired candidate function. Because the series $\sum_{-N}^{N}\left|f_{n}(r)\right|^{2}$ is monotonically increasing and because by Parseval's identity it converges to $\frac{1}{2 \pi} \int_{0}^{2 \pi}\left|f\left(r \mathrm{e}^{\mathrm{i} \theta}\right)\right|^{2} d \theta$, letting $g_{N}(r)=2 \pi \sum_{-N}^{N}\left|f_{n}(r)\right|^{2}$ a straight forward calculation shows that

$$
\int_{0}^{\infty}\left(\int_{0}^{2 \pi}\left|f-g_{N}\right|^{2} d \theta\right)\left(1+r^{2}\right)^{\gamma} r d r=\int_{0}^{\infty}\left[\left(\int_{0}^{2 \pi}\left|f\left(r \mathrm{e}^{\mathrm{i} \theta}\right)\right|^{2} d \theta\right)-g_{N}\right]\left(1+r^{2}\right)^{\gamma} r d r .
$$

Then, by the monotone convergence theorem we may conclude

$$
\left\|f\left(r \mathrm{e}^{\mathrm{i} \theta}\right)-2 \pi \sum_{-N}^{N} f_{n}(r) \mathrm{e}^{\mathrm{i} n \theta}\right\|_{L_{\gamma}^{2}\left(\mathbb{R}^{2}\right)}=\left\|f-g_{N}\right\|_{L_{\gamma}^{2}\left(\mathbb{R}^{2}\right)} \rightarrow 0, \quad \text { as } \quad N \rightarrow \infty,
$$

as desired. Moreover, since for a.e. $r \in[0, \infty)$ the function $f \in H^{s}([0,2 \pi])$, a similar argument can be carried out to show that as $N \rightarrow \infty$ the expressions $\left\|\partial_{\theta}^{\alpha} f\left(r \mathrm{e}^{\mathrm{i} \theta}\right)-2 \pi \sum_{-N}^{N}(i n)^{\alpha} f_{n}(r) \mathrm{e}^{\mathrm{i} n \theta}\right\|_{L_{\gamma}^{2}\left(\mathbb{R}^{2}\right)} \rightarrow 0$ for all integers $\alpha \leq s$. This completes the proof of the lemma.

We also have the following result describing how elements in $M_{\gamma}^{1,2}\left(\mathbb{R}^{d}\right)$ decay at infinity (see Appendix B.1 for a proof).

Lemma 3.2. Given $f \in M_{\gamma}^{1,2}\left(\mathbb{R}^{d}\right)$, then $|f(\mathbf{x})| \leq C\|f\|_{M_{\gamma}^{1,2}\left(\mathbb{R}^{d}\right)}^{d / 2} \cdot\left(1+|\mathbf{x}|^{2}\right)^{-(\gamma+d / 2)}$ as $\mathrm{x} \rightarrow \infty$.

In addition, the next lemma characterizes the multiplication property for Kondratiev spaces. The lemma is more general than we need in the sense that it holds for complete Riemannian manifolds that are euclidean at infinity, ( $\mathbb{M}, e)$. A proof of this result can be found in $\mathrm{CBC} 81$. We have adapted the notation so that it is consistent with our definition of Kondratiev spaces. 
Lemma 3.3. If ( $\mathbb{M}, e)$ is a complete Riemannian manifold euclidean at infinity of dimension $d$, we have the continuous multiplication property

$$
\begin{aligned}
M_{\gamma_{1}}^{s_{1}, 2}(\mathbb{M}) \times M_{\gamma_{2}}^{s_{2}, 2}(\mathbb{M}) & \longrightarrow M_{\gamma}^{s, 2}(\mathbb{M}) \\
\left(f_{1}, f_{2}\right) & \longmapsto f_{1} \cdot f_{2}
\end{aligned}
$$

provided $s_{1}, s_{2} \geq s, s<s_{1}+s_{1}-d / 2$, and $\gamma<\gamma_{1}+\gamma_{2}+d / 2$.

3.1. Fredholm properties of the Laplacian and related operators. The main appeal of Kondratiev spaces for us is that the the Laplace operator is a Fredholm operator in these spaces. This is summarized in the following theorem, whose proof can be found in $\mathrm{McO} 79$. This result is the basis for deriving Fredholm properties for other linear operators that will be encountered in Section 4

Theorem 2. Let $1<p=\frac{q}{q-1}<\infty, d \geq 2$, and $\gamma \neq d / q+m$ or $\gamma \neq 2-d / p-m$, for some $m \in \mathbb{N}$. Then

is a Fredholm operator and

$$
\Delta: M_{\gamma-2}^{2, p}\left(\mathbb{R}^{d}\right) \rightarrow L_{\gamma}^{p}\left(\mathbb{R}^{d}\right),
$$

(1) for $2-d / p<\gamma<+d / q$ the map is an isomorphism;

(2) for $d / q+m<\gamma<d / q+m+1, m \in \mathbb{N}$, the map is injective with closed range equal to

$$
R_{m}=\left\{f \in L_{\gamma}^{p}: \int f(y) H(y)=0 \text { for all } H \in \bigcup_{j=0}^{m} \mathcal{H}_{j}\right\}
$$

(3) for $2-d / p-m-1<\gamma<2-d / p-m, m \in \mathbb{N}$, the map is surjective with kernel equal to

$$
N_{m}=\bigcup_{j=0}^{m} \mathcal{H}_{j}
$$

Here, $\mathcal{H}_{j}$ denote the harmonic homogeneous polynomials of degree $j$.

On the other hand, if $\gamma=2-d / p-m$ or $\gamma=d / q+m$ for some $m \in \mathbb{N}$, then $\Delta$ does not have closed range.

Notice that we can use the result from Lemma 3.1 to diagonalize the Laplacian. That is, given $u \in M_{\gamma-2}^{2,2}\left(\mathbb{R}^{2}\right)$,

$$
\Delta u=\Delta\left(\sum_{n \in \mathbb{Z}} u_{n}(r) \mathrm{e}^{\mathrm{i} i \theta}\right)=\sum_{n \in \mathbb{Z}}\left(\partial_{r r} u_{n}+\frac{1}{r} \partial_{r} u_{n}-\frac{n^{2}}{r^{2}} u_{n}\right) \mathrm{e}^{\mathrm{i} n \theta}=\sum_{n \in \mathbb{Z}}\left(\Delta_{n} u_{n}\right) \mathrm{e}^{\mathrm{i} n \theta},
$$

where $u_{n} \in M_{r, \gamma-2}^{2,2}\left(\mathbb{R}^{2}\right)$.

One can now combine this decomposition together with Theorem 2 to arrive at the following lemma.

Lemma 3.4. Let $\gamma \in \mathbb{R} \backslash \mathbb{Z}$, and $n \in \mathbb{Z}$. Then, the operator $\Delta_{n}: M_{r, \gamma-2}^{2,2}\left(\mathbb{R}^{2}\right) \rightarrow$ $L_{r, \gamma}^{2}\left(\mathbb{R}^{2}\right)$ given by

is a Fredholm operator and,

$$
\Delta_{n} \phi=\partial_{r r} \phi+\frac{1}{r} \partial_{r} \phi-\frac{n^{2}}{r^{2}} \phi
$$

(1) for $1-n<\gamma<n+1$, the map is invertible;

(2) for $\gamma>n+1$, the map is injective with cokernel spanned by $r^{n}$;

(3) for $\gamma<1-n$, the map is surjective with kernel spanned by $r^{n}$.

On the other hand, the operator is not Fredholm for integer values of $\gamma$. 
The next lemma requires that we specify some notation. In this paper we use the symbol $W_{\sigma}^{s, p}$ to denote the space of locally summable, $s$ times weakly differentiable functions $u: \mathbb{R}^{d} \rightarrow \mathbb{R}$ endowed with the norm

$$
\|u\|_{W_{\sigma}^{s, p}\left(\mathbb{R}^{d}\right)}^{p}=\sum_{|\alpha| \leq s}\left\|D^{\alpha} u(\mathbf{x}) \cdot\left(1+|\mathbf{x}|^{2}\right)^{\sigma / 2}\right\|_{L^{p}\left(\mathbb{R}^{d}\right)}^{p} .
$$

Then, we write $W_{r, \sigma}^{s, p}$ to denote the subspace of radially symmetric functions in $W_{\sigma}^{s, p}$. With this notation we can summarize the Fredholm properties of the operator $\mathscr{L}_{\lambda}$, defined in the next lemma.

Lemma 3.5. Given $\gamma \in \mathbb{R}, \lambda \in[0, \infty)$, and $p \in(1, \infty)$, the operator $\mathscr{L}_{\lambda}: \mathcal{D} \longrightarrow$ $L_{r, \gamma}^{p}\left(\mathbb{R}^{2}\right)$ defined by

$$
\mathscr{L}_{\lambda} \Phi=\partial_{r r} \Phi+\frac{1}{r} \partial_{r} \Phi-2 \lambda \partial_{r} \Phi
$$

and with domain $\mathscr{D}=\left\{u \in M_{r, \gamma-1}^{1, p}\left(\mathbb{R}^{2}\right) \mid \partial_{r} u \in W_{r, \gamma}^{1, p}\left(\mathbb{R}^{2}\right)\right\}$, is Fredholm for $\gamma \neq 1-2 / p$. Moreover,

- it is invertible for $\gamma>1-2 / p$, and

- it is surjective with $\mathrm{Ker}=\{1\}$ for $\gamma<1-2 / p$.

Proof. The result follows from Proposition B.6 and Lemma B.5 in Appendix B.3. which show that the operator $\mathcal{L}_{\omega}=\left(\partial_{r}+\frac{1}{r}-2 \lambda\right) \partial_{r}$ is the composition of a Fredholm index $i=0$ operator, $\partial_{r}: M_{r, \gamma-1}^{1, p}\left(\mathbb{R}^{2}\right) \rightarrow M_{r, \gamma}^{1, p}\left(\mathbb{R}^{2}\right)$, and an invertible operator, $\left(\partial_{r}+\frac{1}{r}-2 \lambda\right): W_{r, \gamma}^{1, p}\left(\mathbb{R}^{2}\right) \rightarrow L_{r, \gamma}^{p}\left(\mathbb{R}^{2}\right)$.

Lastly, we include the following proposition whose proof can be found in Appen$\operatorname{dix}$ B.2.

Proposition 3.6. Let $\gamma \in \mathbb{R}, \alpha, \beta \in \mathbb{Z}^{+} \cup\{0\}, m, d \in \mathbb{Z}^{+}$, and $l \in \mathbb{Z}$. Then, the operator,

$$
\Delta^{m}(\mathrm{Id}-\Delta)^{-l}: \mathcal{D} \subset L_{\gamma-2 m}^{p}\left(\mathbb{R}^{d}\right) \longrightarrow L_{\gamma}^{p}\left(\mathbb{R}^{d}\right),
$$

with domain $\mathcal{D}=\left\{u \in L_{\gamma-2 m}^{p}\left(\mathbb{R}^{d}\right) \mid(I d-\Delta)^{-l} u \in M_{\gamma-2 m}^{2 m, p}\left(\mathbb{R}^{d}\right)\right\}$

- is a Fredholm operator for $\alpha+d / p<\gamma<-\beta-d / p+2 m$ with kernel and cokernel given by

$$
\text { Ker }=\bigcup_{j=0}^{\beta} \mathcal{H}_{j, k}, \quad \text { Coker }=\bigcup_{j=0}^{\alpha} \mathcal{H}_{j, k} ;
$$

- and not Fredholm for values of $\gamma \in\{j+d / p: j \in \mathbb{Z}\}$.

\section{Nonlocal Eikonal Equations}

At the outset, we recall our model nonlocal eikonal equation (2) and the hypotheses on the coupling kernels. Our goal for this section is to show the existence of target wave solutions for (2) which bifurcate from the spatially homogeneous solution. We concentrate on the equation

$$
\phi_{t}=\mathcal{L} * \phi-|\mathcal{J} * \nabla \phi|^{2}+\varepsilon g(x, y), \quad(x, y) \in \mathbb{R}^{2},
$$

where again we assume $\mathcal{L}$ is a diffusive kernel that commutes with rotations. As a consequence its Fourier symbol, $L(\mathbf{k})$, is real analytic and a radial function. We also recall the following assumptions. 
Hypothesis. 1.1 The multiplication operator $L$ is a function of $\xi:=|\mathbf{k}|^{2}$. Its domain can be extended to a strip in the complex plane, $\Omega=\mathbb{R} \times\left(-\mathrm{i} \xi_{0}, \mathrm{i} \xi_{0}\right)$ for some sufficiently small and positive $\xi_{0} \in \mathbb{R}$, and on this domain the operator is uniformly bounded and analytic. Moreover, there is a constant $\xi_{m} \in \mathbb{R}$ such that the operator $L(\xi)$ is invertible with uniform bounds for $|\operatorname{Re} \xi|>\xi_{m}$.

Note that, because $L(\xi)$ is analytic, its zeros are isolated.

Hypothesis. 1.2 The multiplication operator $L(\xi)$ has a zero, $\xi^{*}$, of multiplicity $\ell$ which we assume is at the origin. Therefore, the symbol $L(\xi)$ admits the following Taylor expansion near the origin.

$$
L(\xi)=(-\xi)^{\ell}+\mathrm{O}\left(\xi^{\ell+1}\right), \quad \text { for } \quad \xi \sim 0 .
$$

In particular, we pick $\ell=1$.

Hypothesis. 1.3 The kernel $\mathcal{J}$ is radially symmetric, exponentially localized, twice continuously differentiable, and

$$
\int_{\mathbb{R}^{2}} \mathcal{J}(\mathbf{x}) d \mathbf{x}=1
$$

The idea behind our proof for the existence of target wave solutions is to first show that the convolution operator $\mathcal{L}$ behaves much like the Laplacian when viewed in the setting of Kondratiev spaces. In other words, both operators have the same Fredholm properties. Consequently it is possible to precondition equation (2) by an appropriate operator, $\mathscr{M}$, with average one, and obtain an expression which has the Laplacian as its linear part,

$$
\mathscr{M} * \partial_{t} \phi=\Delta \phi-\mathscr{M} *|\mathcal{J} * \nabla \phi|^{2}+\varepsilon \mathscr{M} * g .
$$

We then proceed in a similar manner as in Section 2 and look for solutions of the form, $\phi(x, y, t)=\tilde{\phi}(x, y)-\omega t$. Dropping the tildes from our notation we arrive at

$$
-\omega=\Delta \phi-\mathscr{M} *|\mathcal{J} * \nabla \phi|^{2}+\varepsilon \mathscr{M} * g .
$$

We will look at the following two problems.

(1) Finding a solution to the intermediate approximation, described by equation (10) with the value of the frequency, $\omega$, equal to zero.

(2) Finding a solution valid on the whole domain described again by equation 10, but where we let $\omega=\lambda^{2}$ be a non-negative parameter.

These two solutions are matched, and then the results of Theorem 1 are shown.

This section is organized as follows. In the next subsection we will derive Fredholm properties for the convolution operator $\mathcal{L}$, as well as mapping properties for a number of related convolution operators. Then in Sections 4.2 and 4.3 we prove the existence of solutions to the intermediate approximation and to the full problem, respectively. Finally, in Section 4.4 we prove Theorem 1.

4.1. Nonlocal Operators. The following proposition is the 2-dimensional version of the results form [JSW17, but for convolution kernels with radial symmetry. The results below follow very closely the proofs outlined in Ref. [JSW17, and we include them for the sake of completeness. The proof shows that, with the Hypothesis 1.1 and the more general version of Hypothesis 1.2 in which we assume $\ell \in \mathbb{N}$, the convolution operator $\mathcal{L}$ has the same Fredholm properties as the operator $\Delta^{\ell}$. 
Proposition 4.1. Let $\gamma \in \mathbb{R}, \alpha, \beta \in \mathbb{N} \cup\{0\}$, and suppose the convolution operator $\mathcal{L}: L_{\gamma-2}^{2}\left(\mathbb{R}^{2}\right) \rightarrow L_{\gamma}^{2}\left(\mathbb{R}^{2}\right)$ satisfies Hypothesis 1.1 , and Hypothesis 1.2 with $\ell \in \mathbb{N}$. Then, with appropriate domain $\mathcal{D}$ and

- for $\alpha+2 / p<\gamma<-\beta-2 / p+2 \ell, \mathcal{L}$ is a Fredholm operator with kernel and cokernel given by

$$
\text { Ker }=\bigcup_{j=0}^{\beta} \mathcal{H}_{j, k} \quad \text { Coker }=\bigcup_{j=0}^{\alpha} \mathcal{H}_{j, k}
$$

- whereas for $\gamma=\{j+2 / p: j \in \mathbb{Z}\}, \mathcal{L}$ is not Fredholm.

The above result follows from Proposition 3.6 and Lemmas 4.2 and 4.3 shown below.

Lemma 4.2. Let the multiplication operator, $L(\xi)$, satisfy Hypothesis 1.1, and Hypothesis 1.2 with $\ell \in \mathbb{N}$. Then $L(\xi)$ admits the following decomposition:

$$
L(\xi)=M_{L}(\xi) L_{N F}(\xi)=L_{N F}(\xi) M_{R}(\xi),
$$

where $L_{N F}(\xi)=(-\xi)^{\ell} /(1+\xi)^{\ell}$, and $M_{L / R}(\xi)$ and their inverses are analytic and uniformly bounded on $\Omega$.

Proof. We will just show the result only for $M_{L}(\xi)$ since a similar argument holds for $M_{R}(\xi)$. Let

$$
M_{L}(\xi)=\left\{\begin{array}{ccc}
L(\xi) L_{N F}^{-1}(\xi) & \text { for } & \xi \neq 0 \\
\lim _{\xi \rightarrow 0} L(\xi) L_{N F}^{-1}(\xi) & \text { for } & \xi=0
\end{array}\right.
$$

Since both, $L_{N F}(\xi)$ and $L(\xi)$, are analytic, uniformly bounded, and invertible on $\Omega \cap\left\{\xi \in \mathbb{C}:|\operatorname{Re} \xi|>\xi_{m}\right\}$, it follows that the same is true for $M_{L}(\xi)$. That $M_{L}(\xi)$ is analytic and bounded invertible near the origin follows from Riemann's removable singularity theorem and the following result,

$$
\lim _{\xi \rightarrow 0} L(\xi) L_{N F}^{-1}(\xi)=\lim _{\xi \rightarrow 0} L(\xi) \frac{(1+\xi)^{\ell}}{(-\xi)^{\ell}}=1 .
$$

We show next that the operator $\mathcal{M}_{L / R}: L_{\gamma}^{2}\left(\mathbb{R}^{2}\right) \longrightarrow L_{\gamma}^{2}\left(\mathbb{R}^{2}\right)$ defined by

$$
\begin{array}{ccc}
L_{\gamma}^{2}\left(\mathbb{R}^{2}\right) & \longrightarrow & L_{\gamma}^{2}\left(\mathbb{R}^{2}\right) \\
u & \longmapsto & \mathcal{F}^{-1}\left(M_{L / R}\left(|\mathbf{k}|^{2}\right) \hat{u}\right),
\end{array}
$$

is an isomorphism.

Lemma 4.3. The operator $\mathcal{M}_{L / R}: L_{\gamma}^{2}\left(\mathbb{R}^{2}\right) \longrightarrow L_{\gamma}^{2}\left(\mathbb{R}^{2}\right)$ with Fourier symbol $M_{L / R}\left(|\mathbf{k}|^{2}\right)$ is an isomorphism for all $\gamma \in \mathbb{R}$.

Proof. We first show that $\mathcal{M}_{L / R}$ are bounded from $L_{\gamma}^{2}\left(\mathbb{R}^{2}\right)$ to itself for values of $\gamma \in \mathbb{N} \cap\{0\}$. Indeed this follows from Plancherel's Theorem and the results of Lemma 4.2 Given $u \in L_{\gamma}^{2}\left(\mathbb{R}^{2}\right)$ we find that

$$
\left\|\mathcal{M}_{L / R} u(\mathbf{x})\right\|_{L_{\gamma}^{2}}=\left\|M_{L / R} \hat{u}(\mathbf{k})\right\|_{H^{\gamma}} \leq C\left(\|M\|_{C^{\gamma}}\right)\|\hat{u}(\mathbf{k})\|_{H^{\gamma}}=C\left(\|M\|_{C^{\gamma}}\right)\|u(\mathbf{x})\|_{L_{\gamma}^{2}} .
$$

A similar argument shows that the their inverses, $\mathcal{M}_{L / R}^{-1}: L_{\gamma}^{2}\left(\mathbb{R}^{2}\right) \rightarrow L_{\gamma}^{2}\left(\mathbb{R}^{2}\right)$, are bounded. We can extend this result to values of $\gamma \in \mathbb{Z}^{-}$using duality. Then, because $H^{\gamma}\left(\mathbb{R}^{2}\right)$ is a complex interpolation space between $H^{\lfloor\gamma\rfloor}\left(\mathbb{R}^{2}\right)$ and $H^{\lfloor\gamma\rfloor+1}\left(\mathbb{R}^{2}\right)$, the result holds for all values of $\gamma \in \mathbb{R}$. 
From Lemmas 4.2 and 4.3 it follows that the convolution operator $\mathcal{L}: L_{\gamma}^{2}\left(\mathbb{R}^{2}\right) \rightarrow$ $L_{\gamma}^{2}\left(\mathbb{R}^{2}\right)$ can be decomposed as

$$
\mathcal{L} u=\mathcal{M}_{L} \circ \mathcal{L}_{N F} u=\mathcal{L}_{N F} \circ \mathcal{M}_{R} u
$$

with $\mathcal{L}_{N F} u=(I d-\Delta)^{-\ell} \Delta^{\ell} u$ and $\mathcal{M}_{\mathcal{L} / \mathcal{R}}: L_{\gamma}^{2}\left(\mathbb{R}^{2}\right) \rightarrow L_{\gamma}^{2}\left(\mathbb{R}^{2}\right)$ isomorphisms. Furthermore, both operators, $\mathcal{L}$ and $\mathcal{L}_{N F}$, share the same Fredholm properties. This proves Proposition 4.1

Remark. We note here that the integer $\ell$ that appears in the expression for $\mathcal{L}_{N F}$ is the same integer describing the form of the Fourier symbol $L(\xi)$ in Hypothesis 1.2. In particular, for our problem we have that $\ell=1$, so that for the remainder of the paper we will only consider this case.

We are now in a position to define and describe the mapping properties of a related convolution operator, $\mathscr{M}$ which we will use to precondition equation (2). In terms of the invertible operator $\mathcal{M}_{L}$ defined above, $\mathscr{M}$ is given by

$$
\begin{array}{clc}
\mathscr{M}: H_{\gamma}^{s}\left(\mathbb{R}^{2}\right) & \longrightarrow & H_{\gamma}^{s-2}\left(\mathbb{R}^{2}\right), \\
u & \longmapsto & (\mathrm{Id}-\Delta) \mathcal{M}_{L}^{-1} u
\end{array}
$$

It is clear from the definition that $\mathscr{M} * c=c$ for all constants $c \in \mathbb{R}$.

In addition, we will also need to find mapping properties for, Id $-\mathscr{M}$ and $\mathrm{Id}-J$. These operators will appear in the nonlinearities after preconditioning our equation with $\mathscr{M}$. We start with the next lemma which establishes the boundedness of the operator $\mathscr{M}$, which is straightforward to check.

Lemma 4.4. For $\gamma \in \mathbb{R}$ and $s \in \mathbb{Z}$ the operator $\mathscr{M}: H_{\gamma}^{s}\left(\mathbb{R}^{2}\right) \rightarrow H_{\gamma}^{s-2}\left(\mathbb{R}^{2}\right)$ defined by

$$
\mathscr{M} u=(\operatorname{Id}-\Delta) \mathcal{M}_{L}^{-1} u
$$

is bounded.

Next, we show that in the appropriate spaces the operator Id $-\mathscr{M}$ behaves like the Laplacian operator.

Lemma 4.5. Let $\gamma \in \mathbb{R}$ and define $\mathscr{M}$ as in Lemma 4.4. Then, the convolution operator

$$
\operatorname{Id}-\mathscr{M}: M_{\gamma-2}^{2,2}\left(\mathbb{R}^{2}\right) \rightarrow L_{\gamma}^{2}\left(\mathbb{R}^{2}\right)
$$

is a bounded operator. In particular, the operator is Fredholm with the same Fredholm properties as $\Delta: M_{\gamma-2}^{2,2}\left(\mathbb{R}^{2}\right) \rightarrow L_{\gamma}^{2}\left(\mathbb{R}^{2}\right)$.

Proof. With the notation $\xi=|\mathbf{k}|^{2}$, we look at the Fourier Symbol of Id $-\mathscr{M}$ and decompose it as

$$
\mathcal{F}(\mathrm{Id}-\mathscr{M})(\xi)=\left(1+\xi^{2}\right) M_{L}^{-1}(\xi)\left(\frac{M_{L}(\xi)}{1+\xi^{2}}-1\right)
$$

Since $\left(\frac{M_{L}(\xi)}{1+\xi^{2}}-1\right)$ satisfies Hypothesis 1.1 and Hypothesis 1.2 with $\ell=1$, by Proposition 4.1 we can rewrite it as

$$
\left(\frac{M_{L}(\xi)}{1+\xi^{2}}-1\right)=F_{L} \frac{\xi^{2}}{1+\xi^{2}},
$$

where $F_{L}: H_{\gamma}^{s}\left(\mathbb{R}^{2}\right) \rightarrow H_{\gamma}^{s}\left(\mathbb{R}^{2}\right)$ is an isomorphism. The result of the lemma follows from this decomposition. 
Lastly, we show next that the convolution operator $\operatorname{Id}-\mathcal{J}$ is also a Fredholm operator.

Lemma 4.6. The operator $\operatorname{Id}-\mathcal{J}: \mathcal{D} \rightarrow L_{\gamma}^{2}\left(\mathbb{R}^{2}\right)$, with domain

$$
\mathcal{D}=\left\{u \in L_{\gamma-2}^{2}\left(\mathbb{R}^{2}\right) \mid(\mathrm{Id}-\Delta)^{-1} u \in M_{\gamma-2}^{2,2}\left(\mathbb{R}^{2}\right)\right\},
$$

has the same Fredholm properties as $\Delta(\operatorname{Id}-\Delta)^{-1}: \mathcal{D} \rightarrow L_{\gamma}^{2}\left(\mathbb{R}^{2}\right)$. Moreover, the operator Id $-\mathcal{J}: M_{\gamma-2}^{2,2}\left(\mathbb{R}^{2}\right) \rightarrow H_{\gamma}^{2}\left(\mathbb{R}^{2}\right)$ is bounded.

Proof. Since by hypothesis $\mathcal{J}$ is radially symmetric and exponentially localized it follows that its Fourier symbol, $J(\mathbf{k})$, is a radial function and that $J(\mathbf{k}) \rightarrow 0$ as $|\mathbf{k}| \rightarrow \infty$. Moreover, since its average is 1 , we must also have $J(0)=1$. This implies that $\mathcal{F}(\operatorname{Id}-\mathcal{J})(|\mathbf{k}|)$ satisfies Hypothesis 1.1 and Hypothesis 1.2 with $\ell=1$, and that its Taylor expansion near the origin is $\mathcal{F}(\mathrm{Id}-\mathcal{J})(|\mathbf{k}|) \sim \mathrm{O}\left(|\mathbf{k}|^{2}\right)$. The results of this lemma now follow by Proposition 4.1.

We are now ready to show the existence of solutions for the intermediate approximation and the full solution.

4.2. Intermediate Approximation. In this section we concentrate on the equation

$$
0=\Delta \phi-\mathscr{M} *|\mathcal{J} * \nabla \phi|^{2}+\varepsilon \mathscr{M} * g(x, y), \quad(x, y) \in \mathbb{R}^{2},
$$

which is obtained by preconditioning equation $(2)$ with the operator $\mathscr{M}$. Here, the result $\mathscr{M} * \mathcal{L}=\Delta$ follows from Proposition 4.1 and Lemma 4.4. since the operator $\mathcal{L}$ satisfies Hypothesis 1.1 and Hypothesis 1.2 with $\ell=1$. Our goal is to show existence of solutions to (11) when $g$ is assumed to be an algebraically localized function. More precisely, with the definition for the cut off function, $\chi$, stated in the introduction, we prove the following proposition.

Proposition 4.7. Let $m \in \mathbb{N}$ and suppose $g$ is a localized function in the space $L_{\sigma}^{2}\left(\mathbb{R}^{2}\right)$, with weight strength $\sigma \in(m+1, m+2)$. Then, there exists an $\varepsilon_{0}>0$ and a $C^{1}$ map

$$
\phi:\left(-\varepsilon_{0}, \varepsilon_{0}\right) \quad \longrightarrow \quad M_{\gamma-2}^{2,2}\left(\mathbb{R}^{2}\right) \times \mathbb{R}
$$

with $1<\gamma<2$, that allows us to construct an $\varepsilon$-dependent family of solutions to equation (11). Moreover, this solutions are of the form

$$
\Phi(r, \theta ; \varepsilon)=-\chi(r) \ln \left(1-a_{0} \ln (r)\right)+\varepsilon \sum_{\substack{\alpha=-m \\ \alpha \neq 0}}^{m} a_{\alpha}\left(\frac{\chi(r)}{r^{|\alpha|}}\right) \mathrm{e}^{i \alpha \theta}+\phi(r, \theta ; \varepsilon),
$$

where,

- the constant $a_{0}=\varepsilon a_{0,1}+\mathrm{O}\left(\varepsilon^{2}\right)$, with $a_{0,1}=-\frac{1}{2 \pi} \int_{\mathbb{R}^{2}} g(\mathbf{x}) d \mathbf{x}$;

- for $\alpha \in[-m, m] \cap \mathbb{Z} \backslash\{0\}$ the constants $a_{\alpha}=\frac{1}{2 \pi \alpha} \int_{0}^{\infty} \int_{0}^{2 \pi} \mathscr{M} * g(r, \theta) r^{\alpha} \mathrm{e}^{i \alpha \theta} d \theta r d r$; and

- the function $\phi<C r^{\gamma-1}$ as $r \rightarrow \infty$.

The proof is based on the following ansatz,

$$
\phi(r, \theta ; \varepsilon)=\psi_{0}(r)+\varepsilon \sum_{\substack{n \in \mathbb{Z} \\ n \neq 0}} \psi_{n}(r) \mathrm{e}^{\mathrm{i} \theta n}+\varepsilon \sum_{\substack{\alpha=-m \\ \alpha \neq 0}}^{m} a_{\alpha} C_{\alpha}(r) \mathrm{e}^{i \alpha \theta}+\bar{\phi}(r, \theta ; \varepsilon)
$$


where the function

$$
\psi_{0}(r)=-\chi(r) \ln \left(1-a_{0} \ln (r)\right), \quad a_{0} \in \mathbb{R},
$$

is motivated by the asymptotic analysis of Section 2 In particular, notice that the function $\ln \left(1-a_{0} \ln (r)\right)$ solves the equation $\Delta \psi-\left(\partial_{r} \psi\right)^{2}=0$, and it admits the following series expansion

$$
-\ln \left(1-a_{0} \ln (r)\right)=a_{0} \ln (r)+\frac{1}{2}\left(a_{0} \ln (r)\right)^{2}+\frac{1}{3}\left(a_{0} \ln (r)\right)^{3}+\cdots,
$$

provided $\left|a_{0} \ln (r)\right| \ll 1$. As a result we see that

$$
\Delta\left(\psi_{0}-a_{0} \chi \ln (r)\right)-\left(\partial_{r} \psi_{0}\right)^{2}+\Delta a_{0} \chi \ln (r)=\Delta a_{0} \ln (r)+\text { localized, }
$$

and it is this last equality which proves useful in the simplification of equation (11) and one of the reason why $\psi_{0}$ was picked as we have done.

In addition, we let $C_{\alpha}(r)=\frac{\chi}{r|\alpha|}$ and for the moment we assume that for $n \neq 0$ the pair $\left(\psi_{n}, a_{n}\right) \in M_{r, \sigma-2}^{2,2}\left(\mathbb{R}^{2}\right) \times \mathbb{R}$ solves the equations for the first order approximation to equation (11),

$$
\begin{array}{rr}
\partial_{r r} \psi_{n}+\frac{1}{r} \partial_{r} \psi_{n}-\frac{n^{2}}{r^{2}} \psi_{n}+a_{n} \Delta_{n} C_{n}+\tilde{g}_{n}(r)=0 & \text { for } \quad 0<|n| \leq m \\
\partial_{r r} \psi_{n}+\frac{1}{r} \partial_{r} \psi_{n}-\frac{n^{2}}{r^{2}} \psi_{n}+\tilde{g}_{n}(r)=0 & \text { for } \quad m<|n| .
\end{array}
$$

Here, the inhomogeneous terms $\tilde{g}_{n}$, for $n \in \mathbb{Z}$, are just the polar modes of $\tilde{g}=\mathscr{M} * g$ obtained using Lemma 3.1 .

We now wish to look at the operator $F: M_{\gamma-2}^{2,2}\left(\mathbb{R}^{2}\right) \times \mathbb{R} \times \mathbb{R} \rightarrow L_{\gamma}^{2}\left(\mathbb{R}^{2}\right)$, obtained by inserting the ansatz (12) into equation (11). A straight forward calculation using the results from Lemma 3.1 and the choice of $\psi_{0}$ shows that

$$
\begin{aligned}
F\left(\bar{\phi}, a_{0} ; \varepsilon\right) & =\Delta \bar{\phi}+a_{0} \Delta \chi \ln (r)+\varepsilon \tilde{g}_{0}+P(\bar{\phi}), \\
P(\bar{\phi}) & =-\mathscr{M} *|\mathcal{J} * \nabla \phi|^{2}+\left|\nabla \psi_{0}\right|^{2}+\left(\Delta \psi_{0}-a_{0} \Delta \chi \ln (r)-\left|\nabla \psi_{0}\right|^{2}\right) .
\end{aligned}
$$

The dependence on $\bar{\phi}$ in the definition of the nonlinearity $P$ comes through the function $\phi$, which is just the ansatz (12).

To show the results of Proposition 4.7 we apply the implicit function theorem to find the zeros of $F$. It is easy to check that $F$ depends smoothly on $\varepsilon$, so that we are left with showing three things:

(1) The equations (13) and (14) are invertible.

(2) The operator $F: M_{\gamma-2}^{2,2}\left(\mathbb{R}^{2}\right) \times \mathbb{R} \times \mathbb{R} \rightarrow L_{\gamma}^{2}\left(\mathbb{R}^{2}\right)$ is well defined for $1<\gamma<2$.

(3) The derivative $\left.D_{\bar{\phi}, a_{0}} F\right|_{\varepsilon=0}=\mathscr{L}$ defined as $\mathscr{L}: M_{\gamma-2}^{2,2}\left(\mathbb{R}^{2}\right) \times \mathbb{R} \rightarrow L_{\gamma}^{2}\left(\mathbb{R}^{2}\right)$,

$$
\mathscr{L}(\phi, a)=\Delta \phi+a \Delta \chi \ln (r)
$$

is invertible.

We start with item (11). From Lemma 3.4 we know that in the setting of radial Kondratiev spaces and for $n \in \mathbb{N} \cup\{0\}$ the operators,

$$
\begin{array}{clc}
\Delta_{n}: M_{r, \sigma-2}^{2,2}\left(\mathbb{R}^{2}\right) & \longrightarrow & L_{r, \sigma}^{2}\left(\mathbb{R}^{2}\right), \\
\phi & \longmapsto & \partial_{r r} \phi+\frac{1}{r} \partial_{r} \phi-\frac{n^{2}}{r^{2}} \phi
\end{array}
$$

are invertible for values of $\sigma \in(1-n, 1+n)$ and are Fredholm index $i=-1$ for values of $\sigma>n+1$. It is for this reason, and our assumption that $m+1<\sigma<m+2$, that we have added correction terms, $C_{\alpha}(r)$, only for values of $0<|n| \leq m$. In particular, we 
show in the next lemma that the functions $\Delta_{n} C_{n}$ span the cokernel of the operator (15), so that by an application of Lyapunov Schmidt reduction it follows that the equations, 13 and (14), are solvable and that the value of the constants, $a_{n}$, is given by

$$
a_{n}=\frac{1}{n} \int_{0}^{\infty} \tilde{g}_{n}(r) r^{n} r d r, \quad \text { for } \quad 0<|n| \leq m .
$$

Moreover, since the functions $\psi_{n}$ are in $M_{r, \sigma-2}^{2,2}\left(\mathbb{R}^{2}\right)$ for values of $\sigma \in(m+1, m+2)$, the results from Lemma 3.2 imply that these functions satisfy $\left|\psi_{n}\right|<C r^{-2 m}$ for large values of $r$.

Lemma 4.8. Let $n \in \mathbb{N}$, take $\gamma>1+n$, and define $\Delta_{n}=\partial_{r r}+\frac{1}{r} \partial_{r}+\frac{n}{r^{2}}$. Then, the operator $\mathcal{L}_{n}: M_{r, \gamma-2}^{2,2}\left(\mathbb{R}^{2}\right) \times \mathbb{R} \rightarrow L_{r, \gamma}^{2}\left(\mathbb{R}^{2}\right)$ defined as

$$
\mathcal{L}_{n}(\phi, a)=\Delta_{n} \phi+a \Delta_{n}\left(\frac{\chi}{r^{|n|}}\right)
$$

is invertible.

Proof. This is a consequence of Lemma 3.4 and the fact that the function $\Delta_{n}\left(\frac{\chi}{r^{|n|}}\right)$ spans the cokernel of $\Delta_{n}: M_{r, \gamma-2}^{2,2}\left(\mathbb{R}^{2}\right) \longrightarrow L_{r, \gamma}^{2}\left(\mathbb{R}^{2}\right)$, for $n \in \mathbb{N}$. Indeed, a short calculation shows that

$$
\int_{0}^{\infty} \Delta_{n}\left(\frac{\chi}{r^{|n|}}\right) r^{n} r d r=-n
$$

Next, we show that the operator $F$ is well defined.

Lemma 4.9. Let $\gamma \in(1,2)$, then the operator $P: M_{\gamma-2}^{2,2}\left(\mathbb{R}^{2}\right) \rightarrow L_{\gamma}^{2}\left(\mathbb{R}^{2}\right)$ given by

$$
P(\bar{\phi})=-\mathscr{M} *|\mathcal{J} * \nabla \phi|^{2}+\left|\nabla \psi_{0}\right|^{2}+\left(\Delta \psi_{0}-a_{0} \Delta \chi \ln (r)-\left|\nabla \psi_{0}\right|^{2}\right),
$$

and with $\phi(r, \theta ; \varepsilon)$ as in 12), is well defined.

Proof. Since $\psi_{0}(r)=-\chi \ln \left(1-a_{0} \ln (r)\right)$, we know that the expression $\left(\Delta \psi_{0}-\right.$ $\left.a_{0} \Delta \chi \ln (r)-\left|\nabla \psi_{0}\right|^{2}\right)$ is localized.

To show that the remaining terms are in $L_{\gamma}^{2}\left(\mathbb{R}^{2}\right)$ we let $\Phi(r, \theta ; \varepsilon)=\phi(r, \theta ; \varepsilon)-\psi_{0}$, and write

$$
\mathscr{M} *|\mathcal{J} * \nabla \phi|^{2}=\mathscr{M} *\left[|\mathcal{J} * \nabla \Phi|^{2}+2(\mathcal{J} * \nabla \Phi) \cdot\left(\mathcal{J} * \nabla \psi_{0}\right)+\left|\mathcal{J} * \nabla \psi_{o}\right|^{2}\right] .
$$

From Hypothesis 1.3 it is not hard to see that the operator $\mathcal{J}$ satisfies,

$$
\mathcal{J}: M_{\gamma}^{s, 2}\left(\mathbb{R}^{2}\right) \longrightarrow\left\{u \in M_{\gamma}^{s, 2}\left(\mathbb{R}^{2}\right) \mid D^{s} u \in H_{\gamma+s}^{2}\left(\mathbb{R}^{2}\right)\right\}, \quad \forall \gamma \in \mathbb{R} .
$$

We can also check that the term $\Phi$ is in the space $M_{\gamma-1}^{1,2}\left(\mathbb{R}^{2}\right)$, which together with the mapping properties of $\mathcal{J}$ and Lemma 3.2 implies that the expression $(\mathcal{J} * \nabla \Phi)$ is bounded and decays as $|x|^{-\gamma}$. As a result the function $|\mathcal{J} * \nabla \Phi|^{2} \in H_{\gamma}^{2}\left(\mathbb{R}^{2}\right)$ and we may conclude from the mapping properties of $\mathscr{M}$ stated in Lemma 4.4 that the term $\mathscr{M} *|\mathcal{J} * \nabla \Phi|^{2}$ is well defined.

Similarly, since $\nabla \psi_{0} \in M_{\tau}^{2,2}\left(\mathbb{R}^{2}\right)$ with $-1<\tau<0$, it follows from Lemma 3.2 and the mapping properties of $\mathcal{J}$ that $\left|\mathcal{J} * \nabla \psi_{0}\right|$ decays as $|x|^{-1}$. Consequently, the function $\left(\mathcal{J} * \nabla \psi_{0}\right) \cdot(\mathcal{J} * \nabla \Phi)$ is in the space $H_{\gamma}^{2}\left(\mathbb{R}^{2}\right)$ and the product $\mathscr{M} *(\mathcal{J} *$ $\left.\nabla \psi_{0}\right) \cdot(\mathcal{J} * \nabla \Phi)$ is well defined. 
Lastly, we look at the expression $\mathscr{M} *\left|\mathcal{J} * \nabla \psi_{0}\right|^{2}-\left|\nabla \psi_{0}\right|^{2}$, and rewrite it as follows

$$
\begin{aligned}
\mathscr{M} *\left|\mathcal{J} * \nabla \psi_{0}\right|^{2}-\left|\nabla \psi_{0}\right|^{2}= & \mathscr{M} *\left[\left|(\mathcal{J}-\mathrm{Id}) * \nabla \psi_{0}\right|^{2}+2(\mathcal{J}-\mathrm{Id}) * \nabla \psi_{0} \cdot \psi_{0}\right] \\
& +(\mathscr{M}-\mathrm{Id}) *\left|\nabla \psi_{0}\right|^{2}
\end{aligned}
$$

Using Lemma 4.6 we see that the term $(\mathcal{J}-$ Id $) * \nabla \psi_{0}$ is in the space $H_{\gamma}^{2}\left(\mathbb{R}^{2}\right)$, which being a Banach algebra implies that the same term squared is in $H_{\gamma}^{2}\left(\mathbb{R}^{2}\right)$. Then, the mapping properties of $\mathscr{M}$ and the boundedness of $\nabla \psi_{0}$ show that the first term of the above expression is well defined. To show that the last term is also in the space $L_{\gamma}^{2}\left(\mathbb{R}^{2}\right)$ we use Lemma 3.3 to check that $\left|\nabla \psi_{0}\right|^{2}$ is in $M_{\tau}^{2,2}\left(\mathbb{R}^{2}\right)$ and then appeal to the results of Lemma 4.5 .

To complete the proof of Proposition 4.7 we need to show item (3). Since the nonlinearities are in the space $L_{\gamma}^{2}\left(\mathbb{R}^{2}\right)$ with $\gamma \in(1,2)$ the results from Theorem 2 show that the operator $\Delta: M_{\gamma-2}^{2,2}\left(\mathbb{R}^{2}\right) \rightarrow L_{\gamma}^{2}\left(\mathbb{R}^{2}\right)$ is injective with cokernel spanned by 1. A short calculation shows that $\int_{\mathbb{R}^{2}} \Delta \chi \ln (r) d x=2 \pi$, from which it follows that the operator $\mathscr{L}: M_{\gamma-2}^{2,2}\left(\mathbb{R}^{2}\right) \times \mathbb{R} \rightarrow L_{\gamma}^{2}\left(\mathbb{R}^{2}\right)$,

$$
\mathscr{L}(\phi, a)=\Delta \phi+a \Delta \chi \ln (r)
$$

is invertible. If we now consider regular expansions for $\bar{\phi}$ and $a_{0}$, and apply Lyapounov Schmidt reduction to the equation

$$
\Delta \bar{\phi}+a_{0} \Delta \chi \ln (r)+\varepsilon \tilde{g}_{0}+P(\bar{\phi})=0,
$$

the result is that $a_{0}=\varepsilon a_{0,1}+\mathrm{O}\left(\varepsilon^{2}\right)$ with

$$
a_{0,1}=-\frac{1}{2 \pi} \int_{\mathbb{R}^{2}} \tilde{g}_{0}=-\frac{1}{2 \pi} \int_{\mathbb{R}^{2}} g .
$$

In addition, using Lemma 3.2 we see that the function $\bar{\phi}<C r^{\gamma-1}$ for large values of $r$. This completes the proof of Proposition 4.7.

4.3. Full solution for fixed $\omega=\lambda^{2}$. The following equation is obtained by preconditioning (2) with the operator $\mathscr{M}$ and then assuming that we have an ansatz $\phi(x, y, t, \varepsilon, \lambda)=\tilde{\phi}(x, y, \varepsilon)-\lambda^{2} t$, where $\lambda$ is another parameter. Dropping the tildes from our notation:

$$
-\lambda^{2}=\Delta \phi-\mathscr{M} *|\mathcal{J} * \nabla \phi|^{2}+\varepsilon \mathscr{M} * g(x, y) \quad(x, y) \in R^{2} .
$$

We are again interested in solutions that bifurcate from the steady state $\phi=0$ when $\varepsilon \neq 0$ and $\lambda>0$. We point out that in our original problem the value of $\lambda^{2}$ depends on $\varepsilon$, since it represents the frequency, $\omega$, of the target waves that emerge from the introduction of a perturbation. In the next subsection we will find the value of $\omega=\lambda^{2}$ by matching the solutions from Section 4.2 with the solutions from Proposition 4.10 stated next.

Proposition 4.10. Suppose $g$ is in the space $L_{\sigma}^{2}\left(\mathbb{R}^{2}\right)$ with $\sigma>0$. Then, there exists numbers $\varepsilon_{0}, \lambda_{0}>0$ and a $C^{1}$ map

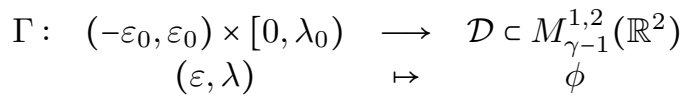


where $\gamma>0$ and $\mathcal{D}=\left\{\phi \in M_{\gamma-1}^{1,2}\left(\mathbb{R}^{2}\right) \mid \nabla \phi \in H_{\gamma}^{1}\left(\mathbb{R}^{2}\right)\right\}$, that allows us to construct an $(\varepsilon, \lambda)$-dependent family of solutions to equation (16). Moreover, these solutions are of the form,

$$
\Phi(r, \theta ; \varepsilon, \lambda)=-\chi(\lambda r) \ln \left(K_{0}(\lambda r)+\phi(r, \theta ; \varepsilon, \lambda),\right.
$$

where

- the function $K_{0}(z)$ is the zero-th order modified Bessel's function of the second kind, and

- the function $\phi<C r^{-\gamma}$ as $r \rightarrow \infty$.

To show the results of the above proposition we again consider an appropriate ansatz motivated by the results from Section 2

$$
\phi(r, \theta)=-\chi(\lambda r) \ln \left(K_{0}(\lambda r)\right)+\bar{\phi}(r, \theta) .
$$

For ease of notation we define $\psi_{0}(r)=-\chi(\lambda r) \ln \left(K_{0}(\lambda r)\right)$ and remark that the function $-\ln \left(K_{0}(\lambda r)\right)$ is a solution to the equation $-\lambda^{2}=\Delta \psi_{0}-\left|\nabla \psi_{0}\right|^{2}$. As a consequence, when inserting the above ansatz into equation 16 we find that $\bar{\phi}$ must satisfy

$$
0=\Delta \bar{\phi}-2 \lambda \partial_{r} \bar{\phi}+\varepsilon \mathscr{M} * g(r, \theta)+P(\bar{\phi}),
$$

where the nonlinear term $P(\bar{\phi})$ is given by

$$
P(\bar{\phi})=-\mathscr{M} *|\mathcal{J} * \nabla \phi|^{2}+\left|\nabla \psi_{0}\right|^{2}+2 \lambda \partial_{r} \bar{\phi}+\left(\lambda^{2}+\Delta \psi_{0}-\left|\nabla \psi_{0}\right|^{2}\right),
$$

and again $\phi$ is as in ansatz (17).

We have chosen to use polar coordinates to highlight the form of the linearization and to take advantage of the polar decomposition of Kondratiev spaces. As in the previous section, we make use of the right hand side of equation 18 as an operator $F: \mathcal{D} \times \mathbb{R} \times \mathbb{R} \rightarrow \mathcal{D}$,

$$
F(\bar{\phi} ; \varepsilon, \lambda)=\operatorname{Id}-\mathscr{L}_{\lambda}^{-1}(\varepsilon \mathscr{M} * g(r, \theta)+P(\bar{\phi})) .
$$

where $\mathcal{D}=\left\{\phi \in M_{\gamma-1}^{1,2}\left(\mathbb{R}^{2}\right) \mid \nabla \phi \in H_{\gamma}^{1}\left(\mathbb{R}^{2}\right)\right\}$, and $\mathcal{L}_{\lambda}$ is defined as $\mathscr{L}_{\lambda}: \mathcal{D} \subset$ $M_{\gamma-1}^{1,2}\left(\mathbb{R}^{2}\right) \longrightarrow L_{\gamma}^{2}\left(\mathbb{R}^{2}\right)$

$$
\mathscr{L}_{\lambda} \phi=\Delta \phi-2 \lambda \partial_{r} \phi, \quad \lambda \geq 0
$$

The results of the proposition then follow by finding the zeroes of $F$ via the implicit function theorem, keeping in mind that the operator is only defined for values of $\lambda \geq 0$. We first notice that $F$ depends smoothly on $\varepsilon$ for all values of $\varepsilon \in \mathbb{R}$, that $F(0 ; 0,0)=0$, and that the Fréchet derivative, $\left.D_{\phi} F\right|_{(\varepsilon, \lambda)=(0,0)}=I$, is invertible. Thus, we are left with showing the following two points:

(1) The operator $F: \mathcal{D} \times \mathbb{R} \times \mathbb{R} \rightarrow \mathcal{D}$ is well defined, and

(2) it is $C^{1}$ with respect to $\lambda \in\left[0, \lambda_{0}\right)$ for some $\lambda_{0}>0$.

The results of item 1.) are true if we can show that the nonlinearities are well defined in $L_{\gamma}^{2}\left(\mathbb{R}^{2}\right)$, and if the linear operator $\mathcal{L}_{\lambda}$ is invertible. The following lemma shows that the first assertion is true.

Lemma 4.11. Let $\gamma>0$, then the operator $P: \mathcal{D} \rightarrow L_{\gamma}^{2}\left(\mathbb{R}^{2}\right)$, defined by

$$
P(\bar{\phi})=-\mathscr{M} *|\mathcal{J} * \nabla \phi|^{2}+\left|\nabla \psi_{0}\right|^{2}+2 \lambda \partial_{r} \bar{\phi}+\left(\lambda^{2}+\Delta \psi_{0}-\left|\nabla \psi_{0}\right|^{2}\right)
$$

and with $\phi(r, \theta ; \varepsilon)$ as in equation 17), is bounded. 
Proof. Because $\psi_{0}=-\chi \ln \left(K_{0}(\lambda r)\right)$, the expression in the parenthesis is localized. To show that the remaining terms are also well defined in the space $L_{\gamma}^{2}\left(\mathbb{R}^{2}\right)$ we write

$$
\mathscr{M} *|\mathcal{J} * \nabla \phi|^{2}=-\mathscr{M} *\left[|\mathcal{J} * \nabla \bar{\phi}|^{2}-2(\mathcal{J} * \nabla \bar{\phi}) \cdot\left(\mathcal{J} * \nabla \psi_{0}\right)+\left|\mathcal{J} * \nabla \psi_{0}\right|^{2}\right] .
$$

Since $\bar{\phi}$ is in the space $\mathcal{D}$ it follows from Hypothesis 1.3 that the term $(\mathcal{J} * \nabla \bar{\phi})$ is in $H_{\gamma}^{3}\left(\mathbb{R}^{2}\right)$, and it is then clear that the expression, $\mathscr{M} *|\mathcal{J} * \nabla \bar{\phi}|^{2}$ is in $L_{\gamma}^{2}\left(\mathbb{R}^{2}\right)$.

Next, we look at the difference

$$
\begin{aligned}
\lambda \partial_{r} \bar{\phi}-\mathscr{M} *\left[(\mathcal{J} * \nabla \bar{\phi}) \cdot\left(\mathcal{J} * \nabla \psi_{0}\right)\right]= & \lambda \partial_{r} \bar{\phi}-\mathscr{M} *\left[\left(\mathcal{J} * \partial_{r} \bar{\phi}\right)\left(\mathcal{J} * \partial_{r} \psi_{0}\right)\right] \\
= & \lambda \partial_{r} \bar{\phi}-\mathscr{M} *\left[\left(\mathcal{J} * \partial_{r} \bar{\phi}\right)\left(\mathcal{J} *\left(\partial_{r} \psi_{0}-\lambda+\lambda\right)\right)\right] \\
& +\lambda \mathcal{J} * \partial_{r} \bar{\phi}-\lambda \mathcal{J} * \partial_{r} \bar{\phi} \\
= & \lambda(\mathcal{J}-\mathrm{Id}) * \partial_{r} \bar{\phi}-\lambda \mathscr{M} *\left(\mathcal{J} * \partial_{r} \bar{\phi}\right) \\
& -\mathscr{M} *\left[\left(\mathcal{J} *\left(\partial_{r} \psi_{0}-\lambda\right)\left(\mathcal{J} * \partial_{r} \bar{\phi}\right)\right)\right],
\end{aligned}
$$

where we used the fact that $\psi_{0}(r)$ is a radial function to do the simplifications. Using Lemmas 4.4 and 4.6 and because $\partial_{r} \bar{\phi}$ is in the space $H_{\gamma}^{2}\left(\mathbb{R}^{2}\right)$, one is able to check that the first two terms in the last equality are well defined functions in $L_{\gamma}^{2}\left(\mathbb{R}^{2}\right)$. The last term is localized since $\partial_{r} \psi_{0}=\frac{\lambda K_{0}^{\prime}(\lambda r)}{K_{0}(\lambda r)}$ approaches $\lambda$ as $r$ goes to infinity, see Table 1 in Section 2 .

Finally, we consider the difference

$$
\begin{aligned}
\mathscr{M} *\left|\mathcal{J} * \nabla \psi_{0}\right|^{2}-\left|\nabla \psi_{0}\right|^{2} & =\mathscr{M} *\left|\mathcal{J} *\left(\partial_{r} \psi_{0}-\lambda+\lambda\right)\right|^{2}-\left(\partial_{r} \psi_{0}\right)^{2} \\
& =\mathscr{M} *\left[\left|\mathcal{J} *\left(\partial_{r} \psi_{0}-\lambda\right)\right|^{2}+2 \lambda \mathcal{J} *\left(\partial_{r} \psi_{0}-\lambda\right)\right]+\lambda^{2}-\left(\partial_{r} \psi_{0}\right)^{2},
\end{aligned}
$$

where in the he last equality we used the fact that $\mathscr{M} * \lambda=\lambda$. Because the function $\partial_{r} \psi_{0}$ approaches $\lambda$ in the far field, It is now easy to see that the above expression is localized.

Our next task is to show that the linear operator $\mathscr{L}_{\lambda}: \mathcal{D} \longrightarrow L_{\gamma}^{2}\left(\mathbb{R}^{2}\right)$ is invertible. To that end we use Lemma 3.1 and decompose $\phi$ into its polar modes,

$$
\mathscr{L}_{\lambda} \phi=\sum_{n \in \mathbb{Z}}\left(\partial_{r r} \phi_{n}+\frac{1}{r} \partial_{r} \phi_{n}-\frac{n^{2}}{r^{2}} \phi_{n}-2 \lambda \partial_{r} \phi_{n}\right) \mathrm{e}^{\mathrm{i} n \theta} .
$$

It follows that $\mathscr{L}_{\lambda}$ is invertible if for each $n \in \mathbb{Z}$ the following operators are also invertible:

$$
\begin{array}{ccc}
\mathscr{L}_{\lambda, n}: \mathcal{D}_{n} & \longrightarrow & L_{r, \gamma}^{2}\left(\mathbb{R}^{2}\right) \\
\phi & \longmapsto & \partial_{r r} \phi+\frac{1}{r} \partial_{r} \phi-\frac{n^{2}}{r^{2}} \phi-2 \lambda \partial_{r} \phi .
\end{array}
$$

Here we used the notation introduced in Section 3 , and defined $\mathcal{D}_{n}=\left\{u \in m_{\gamma-1}^{n}\right.$ | $\left.\partial_{r} u \in L_{r, \gamma}^{2}\left(\mathbb{R}^{2}\right), \partial_{r r} u \in L_{r, \gamma}^{2}\left(\mathbb{R}^{2}\right)\right\}$, so that $\mathcal{D}=\oplus \mathcal{D}_{n}$. Since the multiplication operator $\frac{n^{2}}{r^{2}}: \mathcal{D}_{n} \rightarrow L_{r, \gamma}^{2}\left(\mathbb{R}^{2}\right)$ is compact, the invertibility of $\mathscr{L}_{\lambda, n}$ follows from Lemma 3.5 provided $\gamma>0$. We summarize this result in the following lemma.

Lemma 4.12. Given $\gamma>0$, the operator $\mathscr{L}_{\lambda}: \mathcal{D} \longrightarrow L_{\gamma}^{2}\left(\mathbb{R}^{2}\right)$,

$$
\mathscr{L}_{\lambda} \phi=\Delta \phi-2 \lambda \partial_{r} \phi
$$

is invertible

Finally, to show item 2) we prove that $\mathscr{L}_{\lambda}^{-1}: L_{\gamma}^{2}\left(\mathbb{R}^{2}\right) \rightarrow \mathcal{D}$ depends continuously on the parameter $\lambda$. 
Lemma 4.13. Given $\gamma>0$, the operator $\mathscr{L}_{\lambda}: \mathcal{D} \longrightarrow L_{\gamma}^{2}\left(\mathbb{R}^{2}\right)$,

$$
\mathscr{L}_{\lambda} \phi=\Delta \phi-2 \lambda \partial_{r} \phi
$$

and its inverse, are both $C^{1}$ in $\lambda$ for values of $\lambda \geq 0$.

Proof. That the operator $\mathscr{L}_{\lambda}$ is continuous with respect to the parameter $\lambda$ is straightforward. To show the same result for its inverse we will use the notation $\mathscr{L}_{\lambda}=\mathscr{L}(\lambda)$ to highlight the dependence of the operator on the parameter $\lambda$. At the same time, for $f \in L_{\gamma}^{2}\left(\mathbb{R}^{2}\right)$ and for general $\lambda \geq 0$, we denote by $\phi(\lambda)$ the solution to $\mathscr{L}_{\lambda} \phi=f$ and we look at this next equality

$$
\phi(\lambda+h \lambda)-\phi(\lambda)=-\mathscr{L}(\lambda)^{-1}[\mathscr{L}(\lambda+h \lambda)-\mathscr{L}(\lambda)] \phi(\lambda+h \lambda) .
$$

Because the operator $[\mathscr{L}(\lambda+h \lambda)-\mathscr{L}(\lambda)]$ is bounded from $\mathcal{D}$ to $L_{\gamma}^{2}\left(\mathbb{R}^{2}\right)$ it follows that $\mathscr{L}^{-1}(\lambda)$ is continuous with respect $\lambda$. A short calculation shows that the derivative of $\mathscr{L}^{-1}(\lambda)$ with respect to $\lambda$ is an operator from $L_{\gamma}^{2}\left(\mathbb{R}^{2}\right)$ to $\mathcal{D}$ of the form $\lambda \mathscr{L}^{-1}(\lambda) \partial_{r} \mathscr{L}^{-1}(\lambda)$, and a similar analysis justifies that this operator is indeed continuous with respect to $\lambda$.

Notice that because $\phi \in \mathcal{D} \subset M_{\gamma-1}^{2,2}\left(\mathbb{R}^{2}\right)$, Lemma 3.2 implies that $|\phi|<C r^{-\gamma}$ for large values of $r$. This completes the proof of Proposition 4.10

4.4. Matching and proof of the main theorem. We first find a relation between the frequency, $\omega$, and the parameter $\varepsilon$, and then proceed to show the results from Theorem 1 .

Given $g \in L_{\sigma}^{2}\left(\mathbb{R}^{2}\right)$, with values of $\sigma>1$, we know from Proposition 4.7 that the intermediate solution is of the form

$$
\Phi(r, \theta ; \varepsilon)=-\chi(r) \ln \left(1-a_{0} \ln (r)\right)+\tilde{\phi}(r, \theta ; \varepsilon),
$$

with $\tilde{\phi} \leq r^{-\delta}$, for large $r$ and for values of $\delta \in(0,1)$. At the same time the results from Section 4.3 show that solutions to (16) are given by

$$
\Psi(r, \theta ; \varepsilon, \lambda)=-\chi(\lambda r) \ln \left(K_{0}(\lambda r)\right)+\tilde{\psi}(r, \theta ; \varepsilon, \lambda)
$$

where again $\tilde{\psi} \leq r^{-\delta}$, for values of $\delta>0$ and large $r$.

Since the intermediate approximation is only valid so long as $|\ln (r)| \ll-1 / a_{0}$, and because we are assuming $\lambda$ is small beyond all orders, we may pick the scaling $r=\eta \varepsilon / \lambda$, with $\eta \sim \mathrm{O}(1)$, to do the matching. Notice that in this scaling the function $K_{0}(\lambda r) \sim-\ln (\lambda r / 2)-\gamma_{e}$, where $\gamma_{e}$ is the Euler constant. We can then match the wavenumbers, $\nabla \Phi$ and $\nabla \Psi$, to obtain $\lambda=2 \mathrm{e}^{-\gamma_{e}} \exp \left(-1 / a_{0}\right)$. This in turn gives us an expression for $\omega$ :

$$
\omega=4 \mathrm{e}^{-2 \gamma_{e}} \exp \left(-2 / a_{0}\right) \sim 4 C(\varepsilon) \mathrm{e}^{-2 \gamma_{e}} \exp \left(\frac{4 \pi}{\varepsilon \int_{\mathbb{R}^{2}} g}\right),
$$

where we use the approximation $a_{0}=a_{0,1} \varepsilon+a_{0,2} \varepsilon^{2}+\mathrm{O}\left(\varepsilon^{3}\right)$, with $a_{0,1}=-\frac{1}{2 \pi} \int_{\mathbb{R}^{2}} g$ so that

$$
C(\varepsilon)=\exp \left(\frac{2}{\varepsilon a_{0,1}}-\frac{2}{a_{0}}\right)=\exp \left(\frac{2 C^{\prime}}{a_{0,1}^{2}}\right)+\mathrm{O}(\varepsilon)
$$

Remark. Notice that: 
(1) The above expression shows that if $\varepsilon \int_{\mathbb{R}^{2}} g(x) d x<0$ the frequency $\omega$ is smaller than $\varepsilon$ beyond all orders, which is consistent with our assumptions. In addition, $\omega(\varepsilon)$ depends smoothly on $\varepsilon$ on the interval $\varepsilon \in(0, \infty)$, and by defining $\omega(0)=\partial_{\varepsilon} \omega(0)=0$ we may also conclude that $\omega(\varepsilon)$ is continuously differentiable with respect to $\varepsilon$ on the closed interval $[0, \infty)$.

(2) In Section 2 we derived the following approximation for the frequency:

$$
\omega \sim \frac{4 \mathrm{e}^{-2 \gamma_{e}}}{r_{c}^{2}} \exp \left(\frac{4 \pi}{\varepsilon \int_{\mathbb{R}^{2}} g}\right) .
$$

The constant $r_{c}$ that appears in this expression is therefore accounted for by the constant $C(\varepsilon)$ in 19 .

We can now prove our primary result, Theorem 1, for the nonlocal eikonal equation

$$
\phi_{t}=\mathcal{L} * \phi-|\mathcal{J} * \nabla \phi|^{2}+\varepsilon g(x, y) \quad(x, y) \in \mathbb{R}^{2} .
$$

Theorem 1. Suppose that the kernels $\mathcal{L}$ and $\mathcal{J}$ satisfy Hypotheses 1.1. 1.2 with $\ell=1$, and 1.3. Additionally, suppose $g$ is in the space $L_{\sigma}^{2}\left(\mathbb{R}^{2}\right)$ with $\sigma>1$ and let $M=\frac{1}{2 \pi} \int_{\mathbb{R}^{2}} g<0$. Then, there exists a number $\varepsilon_{0}>0$ and a $C^{1}$ map

$$
\begin{array}{ccc}
\Gamma:\left[0, \varepsilon_{0}\right) & \longrightarrow & \mathcal{D} \subset M_{\gamma-1}^{2,1}\left(\mathbb{R}^{2}\right) \\
\varepsilon & \longmapsto & \psi
\end{array}
$$

where $\gamma>0$ and $\mathcal{D}=\left\{\phi \in M_{\gamma-1}^{1,2}\left(\mathbb{R}^{2}\right) \mid \nabla \phi \in H_{\gamma}^{1}\left(\mathbb{R}^{2}\right)\right\}$, that allows us to construct an $\varepsilon$-dependent family of target pattern solutions to (2). Moreover, these solutions have the form

$$
\Phi(r, \theta, t ; \varepsilon)=-\chi(\lambda(\varepsilon) r) \ln \left(K_{0}(\lambda(\varepsilon) r)\right)+\psi(r, \theta ; \varepsilon)-\lambda^{2}(\varepsilon) t, \quad \lambda(\varepsilon)>0 .
$$

In particular, as $r \rightarrow \infty$,

- $\psi(r ; \varepsilon)<C r^{-\delta}$, for $C \in \mathbb{R}$ and $\delta \in(0,1)$; and

- $\lambda(\varepsilon)^{2} \sim 4 C(\varepsilon) \mathrm{e}^{-2 \gamma_{e}} \exp \left(\frac{2}{\varepsilon M}\right)$, where $C(\varepsilon)$ represents a constant that depends on $\varepsilon$, and $\gamma_{e}$ is the Euler constant.

In addition, these target pattern solutions have the following asymptotic expansion for their wavenumber

$$
k(\varepsilon) \sim \exp \left(\frac{1}{\varepsilon M}\right)+\mathrm{O}\left(\frac{1}{r^{\delta+1}}\right) \quad \text { as } \quad r \rightarrow \infty .
$$

Proof. Considering solutions $\Phi(x, t)=\phi(x)-\lambda^{2} t$ we arrive equation (16). Then, letting

$$
\phi(r, \theta, t ; \varepsilon)=-\chi(\lambda(\varepsilon) r) \ln \left(K_{0}(\lambda(\varepsilon) r)\right)+\psi(r, \theta ; \varepsilon)-\lambda^{2}(\varepsilon) t, \quad \lambda(\varepsilon)>0,
$$

the above analysis together with the matched asymptotics shows the smooth dependence of $\lambda$ on the parameter $\varepsilon$, for $\varepsilon \in(0, \infty)$. We can further define $\lambda$ so that it is continuously differentiable with respect to $\varepsilon$ as $\varepsilon \rightarrow 0$. The same arguments as in Proposition 4.10 can be adapted, but now the nonlinear operator $F$ specified in that proof depends only on $\varepsilon$. Therefore, there exists an $\varepsilon_{0}>0$ so that the above ansatz indeed represents an $\varepsilon$-dependent family of solutions to 16 for $\varepsilon \in\left[0, \varepsilon_{0}\right)$. The theorem follows directly. 


\section{Discussion}

In this paper we derive a model nonlinear integro-differential equation to describe the phase evolution of an array of oscillators with nonlocal diffusive coupling ( see equation (2) and Appendix A). We prove the existence of target wave solutions in such systems when a pacemaker is introduced, which we model as a localized perturbation of the natural frequency of the oscillators. This work builds on earlier results by Doelman et al on phase dynamics for modulated wave trains DSSS05, and on work by Kollár and Scheel on radial patterns generated by inhomogeneities KS07. In contrast to the systems considered in the earlier papers, our model equation accounts for nonlocal interaction and nonradially symmetric perturbations, so our results are applicable to a wider class of systems. However, in other aspects, the earlier results are stronger and they motivate natural directions for future work. In particular:

(1) Starting from a reaction-diffusion system, Doelman et al rigorously prove the validity of the (local) viscous eikonal equation as a reduced, modulational equation DSSS05]. Our nonlocal model (2) generalizes this equation but its derivation in Appendix $\mathrm{A}$ is purely formal. A natural question would be the rigorous validation of our model as a reduction of a nonlocal reaction-diffusion equation, e.g a neural field model.

(2) On the other hand, Kollár and Scheel showed the existence of target wave solutions for the "original" reaction-diffusion system (1), in a situation where the inhomogeneity $g=g(r)$ is a radial function. This motivates proving the existence of target patterns in the "original" neural field or continuum coupled model without appealing to a reduced model of phase dynamics.

(3) Kollár and Scheel also considered the case of perturbations with positive mass, i.e. the "mass" $\varepsilon \int g>0$, and showed the existence of weak sinks and contact defects. The latter can be characterized as waves which propagate towards the core, but with wavenumber that converges to zero at infinity. We conjecture that a similar result should hold in our model (2) for nonradial inhomogeneities and radial convolution kernels (where the origin is a distinguished point). We believe that this case is accessible to the methods presented in this paper.

(4) The case of a mass zero perturbation was considered previously in Sim76. Here, the author shows the existence of a weakly bound ground state with an energy $-\omega \sim-\exp \left(-1 /(c \varepsilon)^{2}\right)$, with $\varepsilon, c>0$. Preliminary analysis indicates that an analogous result is true for our nonlocal eikonal equation (2).

One of our main findings is that nonradially symmetric inhomogeneities generate target patterns which are radially symmetric in the far field. This behavior can be understood heuristically by noticing that when $\varepsilon=0$ the proposed phase equation is invariant under rotations. On the other hand, when $\varepsilon \neq 0$ the inhomogeneity breaks the radial symmetry but only in a region near its core where its presence is not negligible. Our simulations confirm this behavior showing target patterns that are nonradially symmetric near the origin, but that recover this symmetry in the far field. This behavior can also be explained by the analysis. When we decompose our full equation into polar modes (see Section 4.3), the only solution that does not decay algebraically is the one that corresponds to the zero-th mode equation. 
This suggest that what is relevant for the system is the mass of the inhomogeneity and not its shape. This stands in contrasts to the behavior of radially symmetric propagating fronts in bistable reaction-diffusion system for dimensions two or higher Rou04. In this case, nonradially symmetric perturbations to the original front persist and solutions lose their original radial symmetry.

As mentioned in the introduction, the approximations that we have obtained for the frequency of the target patterns are in good agreement with, and in fact refine previous results. However, for values of $\varepsilon$ that we can numerically compute with, they are not accurate enough to enable us to compare the expressions for the frequency in 190 with simulations. In ongoing work, we are developing higher order asymptotic solutions to correctly predict and compare the frequency with numerical experiments.

Finally, the applications that motivated our model equation were large collections of discrete objects, e.g. neurons or particles in a granular medium. Because they involve a large number of interacting units, it seems reasonable to use a continuum model to describe these systems. Our results show that, in general, target wave patterns are found whenever an oscillatory extended system with (possibly nonlocal) diffusive coupling is perturbed by an inhomogeneity (of the appropriate sign). A natural question is to characterize patterns in smaller networks, i.e. systems that cannot be approximated as a continuum. This question involves an extra complication, since the topology of the network now becomes very relevant.

For regular networks, like chains or rings of oscillators with nearest neighbor coupling, it has been shown that a single pacemaker can entrain the whole system RMO06]. A similar result was found for networks where the oscillators are connected at random [KM04. However, in general, the question of understanding the transition to synchrony and the stability of this solution in networks with different topologies is challenging (see [ADGK ${ }^{+} 08$ ] and references therein). For example, in the case of all to all coupling this transition is a function of the coupling strength Kur75, but as connections are removed at random the transition depends now on how many oscillators are still connected $\mathrm{ROH05}$. At the same time, new types of synchronous states called chimeras have been discovered in simulations of oscillators with nonlocal coupling, KB02. These solutions are characterized by having a fraction of the oscillators in phase while the rest are in complete asynchrony (see YHLZ13 and references). A similar behavior was also found in a reaction-diffusion system that can be approximated by an integro-differential equation. In this case, chimera states appear as spiral wave solutions with a core that is not in synchrony SK04. There is a wealth of such physical phenomena that are yet to be analyzed rigorously, and in the future we hope to further investigate aspects of the central question, namely the interplay between discreteness, nonlocal spatial coupling and "local" oscillatory behavior.

\section{ACKNOWLEDGEMENTS}

Both authors would like to thank Arnd Scheel for many useful discussions and suggestions. G.J. acknowledges the support from the National Science Foundation through the grant DMS-1503115. S.V. acknowledges the support from the Simons Foundation through award 524875. 


\section{Appendix A. A hierarchy of eVolution equations for the PHASes of} COUPLED OSCILLATORS

A continuum of oscillators with stable limit cycles and small variations in their natural frequencies can be described by the Stuart-Landau equations Kur84

$$
\partial_{t} z(x, y, t)=i\left(1+\epsilon^{2} g(x, y)\right) z+\left(1-|z|^{2}\right) z
$$

where $\epsilon \ll 1$, and time has been scaled so that the natural frequencies are all close to 1. We assume the coefficient of the nonlinear term $|z|^{2} z$ is real, while full generality allows for a non-zero imaginary part resulting in a dependence of the oscillation frequency on the local amplitude $|z|$ Kur84]. Introducing a non-zero imaginary part for this coefficient will not change any of our conclusions, since $|z| \approx 1$ as we argue below, so the frequency is still nearly constant spatially, just "renormalized" to a different value.

There are indeed many different ways to spatially couple these oscillators. In order to constrain the possible models, we will impose the following requirements:

(1) If $\epsilon=0$, the uniform states $z=e^{i t+\phi_{0}}$ are (neutrally) stable. This is clearly necessary if we hope to describe the $\epsilon \neq 0$ behavior through a slow modulation of the phase, i.e. by replacing the constant $\phi_{0}$ with a slowly varying (in time) function $\phi(x, y, t)$.

(2) The spatial coupling between the oscillators is weak. Quantitatively, we will assume that the coupling is $O(\epsilon)$.

(3) The spatial coupling is isotropic and translation invariant. In particular, this implies that the entire systems is nearly isotropic and translation invariant, and the only place where this symmetry is "weakly" broken is through explicit spatial dependence of the natural frequencies of the oscillators encoded in the term $\epsilon^{2} g(x, y)$.

(4) The overall dynamics has a global gauge symmetry $z(x, y, t) \mapsto e^{i \phi_{0}} z(x, y, t)$ for any constant $\phi_{0}$. This symmetry is appropriate for studying slow behavior in systems with fast oscillations (here the natural frequencies $\approx 1$ for the oscillators correspond to the "fast" time scale) and reflects the fact that the slow behavior is independent of the exact choice of the origin of time (the phase shift $\phi_{0}$ ) if it is governed by averaging over many periods of the fast oscillations [AK02.

Spatial couplings satisfying these requirements are of the form $\epsilon \mathcal{L} \star F\left(|z|^{2}\right) z$, where $\mathcal{L} \star$ denotes convolution with a kernel that is isotropic and $O(1)$ as $\epsilon \rightarrow 0$. Convolutions are manifestly translation invariant and $F\left(|z|^{2}\right) z$ is the general form of a gauge invariant term. Finally, in order to maintain the neutral stability of the uniform states $z=e^{i t+\phi_{0}}$ for $\epsilon=0$, we will need that $\mathcal{L} \star 1=0$, i.e. constants are in the kernel of $\mathcal{L}$. Taken together, this suggests that we should consider models of the form

$\partial_{t} z=i(1+\epsilon g) z+\left(1-|z|^{2}\right) z+\epsilon\left(\mathcal{L}_{1} \star F_{1}\left(|z|^{2}\right) z+\mathcal{L}_{2} \star F_{2}\left(|z|^{2}\right) z+\ldots+\mathcal{L}_{n} \star F_{n}\left(|z|^{2}\right) z\right)$

This is the appropriate generalization of the complex Ginzburg-Landau equation Kur84, AK02 to our context of nonlocally coupled oscillators whose frequencies are amplitude independent. Following the approach in [Kur84, we write $z(x, y, t)=$ $r(x, y, t) e^{i t+i \phi(x, y, t)}$ and expect that $r(x, y, t) \approx 1$ (corresponding to the natural dynamics of the oscillators) and $\left|\partial_{t} \phi\right| \ll 1$ (since we have already accounted the dominant part of the oscillatory behavior $\left.z \sim e^{i t}\right)$. We will call $\phi$ the relative phase 
of the oscillators. While the relative phase varies slowly in time, we make no a priori assumptions about its spatial variation. The above arguments suggest that we can replace $|z|^{2}$ by 1 in the above expressions to get

$$
\begin{aligned}
\mathcal{L} & =F_{1}(1) \mathcal{L}_{1}+F_{2}(1) \mathcal{L}_{2}+\cdots+F_{n}(1) \mathcal{L}_{n} \\
\partial_{t} r+i \partial_{t} \phi & =i \epsilon^{2} g+\left(1-|r|^{2}\right) r+\epsilon e^{-i \phi} \mathcal{L} \star r e^{i \phi}
\end{aligned}
$$

Separating the real and imaginary parts gives the evolution equations for $r$ and $\phi$. Our assumptions imply that the dominant part of the $r$ dynamics is $\partial_{t} r \approx r\left(1-r^{2}\right)$ so that the system relaxes to its slow manifold $r \approx 1$ quickly, i.e on the time scale of the natural oscillations. Indeed, $r$ is slaved to $\phi$ in this regime and we can solve by linearizing $r\left(1-r^{2}\right) \approx-3(r-1)$ near $r=1$ to obtain

$$
r \approx 1+\frac{\epsilon}{3} \operatorname{Re}\left[e^{-i \phi} \mathcal{L} \star e^{i \phi}\right] \approx 1 .
$$

To this order of approximation, the evolution equation for the phase is therefore

$$
\partial_{\tau} \phi=\epsilon g+\operatorname{Im}\left[e^{-i \phi} \mathcal{L} \star e^{i \phi}\right]
$$

where $\tau=\epsilon t$ is the natural slow time that arises from balancing the time derivative of $\phi$ with the nonlocal coupling. This equation can also be recast as $\partial_{\tau} \phi(\mathbf{x})=$ $\epsilon g(\mathbf{x})+\int K_{R}\left(\left|\mathbf{x}-\mathbf{x}^{\prime}\right|\right) \sin \left(\phi\left(\mathbf{x}^{\prime}\right)-\phi(\mathbf{x})\right) d \mathbf{x}^{\prime}+\int K_{I}\left(\left|\mathbf{x}-\mathbf{x}^{\prime}\right|\right) \cos \left(\phi\left(\mathbf{x}^{\prime}\right)-\phi(\mathbf{x})\right) d \mathbf{x}^{\prime}$ by splitting into real and imaginary parts. This is a variant of a well known equation in the context of neural field models (see [SL12] and references therein).

We now specialize 20 to particular types of spatial coupling $\mathcal{L}$. The class of translation invariant, isotropic, differential operators of finite order that annihilate the constants are given by

$$
\mathcal{L} \star z=\left(a_{1}+i b_{1}\right) \Delta z+\left(a_{2}+i b_{2}\right) \Delta^{2} z+\cdots+\left(a_{n}+i b_{n}\right) \Delta^{n} z
$$

where $\Delta=\partial_{x}^{2}+\partial_{y}^{2}$ is the Laplacian. This motivates the definitions

$$
\Gamma_{k}[\phi]=\operatorname{Im}\left[e^{-i \phi} \Delta^{k} e^{i \phi}\right], \quad \Sigma_{k}[\phi]=\operatorname{Re}\left[e^{-i \phi} \Delta^{k} e^{i \phi}\right], \quad k=1,2,3, \ldots
$$

In terms of these operators, the slow evolution of the relative phase $\phi$ is given by

$$
\partial_{\tau} \phi=\epsilon g+a_{1} \Gamma_{1}[\phi]+b_{1} \Sigma_{1}[\phi]+\cdots+a_{n} \Gamma_{n}[\phi]+b_{n} \Sigma_{n}[\phi]
$$

These evolution equations, for different choices of $a_{i}, b_{i}$, are universal models for the slow time evolution of the relative phases of coupled oscillators. We can compute the first few $\Gamma_{k}$ and $\Sigma_{k}$ explicitly to obtain

$$
\begin{aligned}
& \Gamma_{1}[\phi]=\Delta \phi \\
& \Sigma_{1}[\phi]=-|\nabla \phi|^{2} \\
& \Gamma_{2}[\phi]=\Delta^{2} \phi-2|\nabla \phi|^{2} \Delta \phi-4 \nabla \phi \cdot \nabla \nabla \phi \nabla \phi \\
& \Sigma_{2}[\phi]=|\nabla \phi|^{4}-4 \nabla \phi \cdot \nabla(\Delta \phi)-2|\nabla \nabla \phi|^{2}-(\Delta \phi)^{2}
\end{aligned}
$$

where $\nabla \nabla \phi$ is the $2 \times 2$ Hessian matrix. The expressions for $\Gamma_{k}$ and $\Sigma_{k}$ quickly get very complicated as $k$ increases,. We therefore record a few observations on the structure of $\Gamma_{k}$ and $\Sigma_{k}$ that are easy to show by an inductive argument using the recursion $\Sigma_{k}+i \Gamma_{k}=e^{-i \phi} \Delta\left[\left(\Sigma_{k-1}[\phi]+i \Gamma_{k-1}[\phi]\right) e^{i \phi}\right]$

(1) Every term in $\Gamma_{k}$ and $\Sigma_{k}$ has a total of $2 k$ spatial derivatives of $\phi$.

(2) $\Gamma_{k}$ has terms that are homogeneous with degrees $1,3, \ldots, 2 k-1$ in $\phi, \nabla \phi, \nabla \nabla \phi, \ldots$ and $\Sigma_{k}$ has terms that are homogeneous with degrees $2,4, \ldots, 2 k$. 
(3) $\Gamma_{k}[\phi]=\Delta^{k} \phi+$ h.o.t, $\Sigma_{k}[\phi]$ is an isotropic sum/difference of squares of $k$ th order spatial derivatives of $\phi+$ h.o.t, where h.o.t. refers to terms that are higher order in the homogeneity in $\phi$.

We can obtain the dispersion relation by substituting a plane wave ansatz $\phi=$ $k_{x} x+k_{y} y-\omega\left(k_{x}, k_{y}\right) \tau$ into (21) with $\epsilon=0$ to obtain

$$
\omega=b_{1} k^{2}-b_{2} k^{4}+\cdots
$$

where $k^{2}=k_{x}^{2}+k_{y}^{2}$. We will henceforth restrict ourselves to the case where the longwave dispersion coefficient $\left.\frac{d^{2} \omega}{d k^{2}}\right|_{k=0}=2 b_{1}>0$. We define a hierarchy of evolution equations by retaining the linear and quadratic terms in $\Gamma_{k}$ and $\Sigma_{k}$. The first two equations in this hierarchy are

$$
\begin{aligned}
& \partial_{\tau} \phi=\epsilon g+a_{1} \Delta \phi-b_{1}|\nabla \phi|^{2} \\
& \partial_{\tau} \phi=\epsilon g+a_{1} \Delta \phi+a_{2} \Delta^{2} \phi-b_{1}|\nabla \phi|^{2}-b_{2}\left(2|\nabla \nabla \phi|^{2}+(\Delta \phi)^{2}\right)
\end{aligned}
$$

A basic consistency check for our procedure is that the first equation in the hierarchy is indeed the usual viscous eikonal equation.

One method in modeling physical phenomena is to retain just as many terms in the model as necessary to obtain the desired behavior in the solutions. Here, we take a slightly different modeling approach. Our goal is to get the right small $k$ (long-wavelength) behavior. To this end, we will approximate the quadratic terms involving the sums/differences of squares by a single term of the form $-|\mathcal{J} * \nabla \phi|^{2}$ that captures the long-wave behavior of these nonlinearities. That is, we will require that, for an exponent $\alpha$ that is as large as possible, and for all compactly supported smooth function $\psi \geq 0$, we have

$$
\int\left\{b_{1}|\nabla \phi|^{2}+b_{2}\left(2|\nabla \nabla \phi|^{2}+(\Delta \phi)^{2}\right)-|\mathcal{J} * \nabla \phi|^{2}\right\} \psi\left(\frac{x}{L}, \frac{y}{L}\right) d x d y \sim O\left(L^{-\alpha}\right) \text { as } L \rightarrow \infty .
$$

For any function $g \in L^{1}\left(\mathbb{R}^{2}\right)$, the dominated convergence theorem implies that, as $L \rightarrow \infty, \int g(x, y) \psi\left(\frac{x}{L}, \frac{y}{L}\right) d x d y \rightarrow \psi(0) \hat{g}(0)$ where $\hat{g}=\mathcal{F}(g)$ denotes the Fourier transform of $g$. Consequently, for $\phi \in C_{0}\left(\mathbb{R}^{2}\right)$, our requirement that the approximation should capture the right long-wave behavior of the nonlinearity can be written as

$$
\mathcal{F}\left(b_{1}|\nabla \phi|^{2}+b_{2}\left(2|\nabla \nabla \phi|^{2}+(\Delta \phi)^{2}\right)\right)(0)=\mathcal{F}\left(|\mathcal{J} * \nabla \phi|^{2}\right)(0)
$$

We now show that we can indeed find an expression for the Fourier symbol corresponding to the convolution operator $\mathcal{J}$ satisfying this condition.

With $\mathbf{k}=\left(k_{1}, k_{2}\right)$ representing our variable in Fourier space and defining the nonlinear operator $N$ by

$$
\begin{aligned}
N(\hat{\phi})= & \mathcal{F}\left(b_{1}\left(\phi_{x}^{2}+\phi_{y}^{2}\right)+2 b_{2}\left(\phi_{x x}^{2}+2 \phi_{x y}^{2}+\phi_{y y}^{2}\right)+b_{2}\left(\phi_{x x}^{2}+2 \phi_{x x} \phi_{y y}+\phi_{y y}^{2}\right)\right) \\
= & -b_{1}\left(k_{1} \hat{\phi} * k_{1} \hat{\phi}+k_{2} \hat{\phi} * k_{2} \hat{\phi}\right)+3 b_{2}\left(k_{1}^{2} \hat{\phi} * k_{1}^{2} \hat{\phi}+k_{2}^{2} \hat{\phi} * k_{2}^{2} \hat{\phi}\right) \\
& \left.+4 b_{2}\left(k_{1} k_{2} \hat{\phi} * k_{1} k_{2} \hat{\phi}\right)+2 b_{2}\left(k_{1}^{2} \hat{\phi} * k_{2}^{2} \hat{\phi}\right)\right),
\end{aligned}
$$


a straight forward computation then shows that

$$
\begin{aligned}
& N(\hat{\phi})(0)=\int_{\mathbb{R}^{2}}\left(b_{1}+3 b_{2}|\mathbf{q}|^{2}\right)|\mathbf{q}|^{2} \hat{\phi}(-\mathbf{q}) \hat{\phi}(\mathbf{q}) d \mathbf{q} \\
& N(\hat{\phi})(0)=\int_{\mathbb{R}^{2}} J(-\mathbf{q}) \hat{\phi}(-\mathbf{q})\left\langle\mathrm{i} q_{1}, \mathrm{i} q_{2}\right\rangle \cdot\left\langle-\mathrm{i} q_{1},-\mathrm{i} q_{2}\right\rangle J(\mathbf{q}) \hat{\phi}(\mathbf{q}) d \mathbf{q}
\end{aligned}
$$

where $J(\mathbf{k})=\sqrt{b_{1}+3 b_{2}|\mathbf{k}|^{2}}$. Since this holds for all $\phi \in C_{0}\left(\mathbb{R}^{2}\right)$, it is clear then that the Fourier symbol for $\mathcal{J}$ can be picked to be precisely the multiplication operator $J(\mathbf{k})$.

An inductive argument allows us to extend these ideas to higher order equations in the hierarchy (cf. (22)), viz. truncating (21) at quadratic order, followed by a reduction of the quadratic terms as above will give a reduced model

$$
\partial_{\tau} \phi=\sum a_{j} \Delta^{j} \phi-|\mathcal{J} * \nabla \phi|^{2}
$$

where the kernel $\mathcal{J}$ has a Fourier symbol of the form

$$
J(\mathbf{k})=\left[\sum_{j} c_{j} b_{j}|\mathbf{k}|^{2(j-1)}\right]^{1 / 2}
$$

and the constants $c_{j}$ are universal (i.e. independent of the differential operator $\mathcal{L}$ ) and can be computed explicitly. In particular, $c_{1}=1, c_{2}=3$.

If we assume that the function $e^{i \phi}$ is band-limited, a physically reasonable assumption precluding the presence of arbitrarily small-scale structures in the pattern of the relative phase, we can extend the above argument to reduce (21) for general (i.e. not necessarily a finite order differential operators) $\mathcal{L}$ and $\mathcal{J}$ with continuous Fourier symbols $L=L(|\mathbf{k}|), J=J(|\mathbf{k}|)$ that are also exponentially localized. Indeed observing that the space $C_{0}\left(\mathbb{R}^{2}\right)$ is dense in weighted Sobolev spaces, an argument by approximation gives a reduction of (21) to

$$
\partial_{\tau} \phi=\epsilon g+\mathcal{L} * \phi-|\mathcal{J} * \nabla \phi|^{2} .
$$

By rescaling $\tau$ and $\phi$ we can, W.L.O.G, set $a_{1}=1, b_{1}=1$, so that $L(0)=1, J(0)=1$. This is the basic model (Eq. (2)) that we consider in the body of the paper.

Appendix B. Proofs of subsidiary Results

\section{B.1. Algebraic decay in Kondratiev spaces.}

Lemma. 3.2. Let $f \in M_{\gamma}^{1,2}\left(\mathbb{R}^{d}\right)$ then $|f(\mathbf{x})| \leq C\|f\|_{M_{\gamma}^{1,2}\left(\mathbb{R}^{d}\right)}\left(1+|\mathbf{x}|^{2}\right)^{-(\gamma+d / 2)}$ as $|\mathbf{x}| \rightarrow \infty$. 
Proof. Let $(\theta, r)$ represent spherical coordinates in $d$ dimensions, with $r$ being the radial direction and $\theta$ representing the coordinates in the unit sphere, $\Sigma$. Then

$$
\begin{aligned}
\int_{\Sigma}|f(\theta, r)|^{2} d \theta & \leq \int_{\Sigma}\left(\int_{\infty}^{R}\left|\partial_{r} f(\theta, s)\right| d s\right)^{2} d \theta \\
& =\int_{\Sigma}\left(\int_{\infty}^{R} s^{\alpha} s^{\gamma+1}\left|\partial_{r} f(\theta, s)\right| s^{(d-1) / 2} d s\right)^{2} d \theta \\
& \leq \int_{\Sigma}\left(\int_{\infty}^{R} s^{2 \alpha}\right)^{2}\left(\int_{\infty}^{R} s^{2(\gamma+1)}\left|\partial_{r} f(\theta, s)\right|^{2} s^{(d-1)} d s\right)^{2} d \theta \\
& \leq R^{2 \alpha+1}\|\nabla f\|_{L_{\gamma+1}^{2}\left(\mathbb{R}^{d}\right)}^{2}
\end{aligned}
$$

where $\alpha=-(\gamma+1)+(1-d) / 2$ and $R$ is fixed. We therefore have the following inequality

$$
\|f(\cdot, R)\|_{L^{2}} \leq R^{-(\gamma+d / 2)}\|\nabla f\|_{L_{\gamma+1}^{2}\left(\mathbb{R}^{d}\right)}
$$

From Adams and Fournier [AF03, Theorem, 5.9] we know that there exist a constant $C$ such that

$$
\|f(\cdot, R)\|_{\infty} \leq C\|f(\cdot, R)\|_{W^{m, p}\left(\mathbb{R}^{d}\right)}^{\delta}\|f(\cdot, R)\|_{L^{q}\left(\mathbb{R}^{d}\right)}^{(1-\delta)}
$$

where $\delta=d p /(d p+(m p-d) q)$ and $1 / q+1 / p=1$. Choosing $p=q=2$ and $m=0$, we obtain

$$
\|f(\cdot, R)\|_{\infty} \leq C\|f(\cdot, R)\|_{L^{2}} \leq C R^{-(\gamma+d / 2)}\|\nabla f\|_{M_{\gamma}^{1,2}\left(\mathbb{R}^{d}\right)}
$$

B.2. Fredholm properties of $\Delta^{m}(\operatorname{Id}-\Delta)^{-l}$. In this section we look at the Fredholm properties of the operator

$$
\Delta^{m}(\operatorname{Id}-\Delta)^{-l}: \mathcal{D} \subset L_{\gamma-2 m}^{p}\left(\mathbb{R}^{d}\right) \quad \longrightarrow \quad L_{\gamma}^{p}\left(\mathbb{R}^{d}\right)
$$

with domain $\mathcal{D}=\left\{u(\mathbf{x}) \in L_{\gamma-2 m}^{p}\left(\mathbb{R}^{d}\right) \mid(\operatorname{Id}-\Delta)^{-l} u(\mathbf{x}) \in M_{\gamma-2 m}^{2 m, p}\left(\mathbb{R}^{d}\right)\right\}$. More precisely, we prove the following proposition.

Proposition. 3.6. Let $\gamma \in \mathbb{R}, \alpha, \beta \in \mathbb{Z}^{+} \cup\{0\}, m, d \in \mathbb{Z}^{+}$, and $l \in \mathbb{Z}$. Then, the operator,

$$
\Delta^{m}(\mathrm{Id}-\Delta)^{-l}: \mathcal{D} \subset L_{\gamma-2 m}^{p}\left(\mathbb{R}^{d}\right) \longrightarrow L_{\gamma}^{p}\left(\mathbb{R}^{d}\right),
$$

with domain $\mathcal{D}=\left\{u \in L_{\gamma-2 m}^{p}\left(\mathbb{R}^{d}\right) \mid(\operatorname{Id}-\Delta)^{-l} u \in M_{\gamma-2 m}^{2 m, p}\left(\mathbb{R}^{d}\right)\right\}$

- is a Fredholm operator for $\alpha+d / p<\gamma<-\beta-d / p+2 m$ with kernel and cokernel given by

$$
\text { Ker }=\bigcup_{j=0}^{\beta} \mathcal{H}_{j, k}, \quad \text { Coker }=\bigcup_{j=0}^{\alpha} \mathcal{H}_{j, k} ;
$$

- and not Fredholm for values of $\gamma \in\{j+d / p: j \in \mathbb{Z}\}$. of

The proof consists in showing that the operator $\Delta^{m}(\mathrm{Id}-\Delta)^{l}$ is the composition

(1) an invertible operator $(\operatorname{Id}-\Delta)^{-l}: \mathcal{D} \subset L_{\gamma-2}^{p}\left(\mathbb{R}^{d}\right) \longrightarrow M_{\gamma-2 m}^{2 m, p}\left(\mathbb{R}^{d}\right) \subset W_{\gamma-2 m}^{2 m, p}\left(\mathbb{R}^{d}\right)$ 
(2) and a Fredholm operator $\Delta^{m}: M_{\gamma-2}^{2 m, p}\left(\mathbb{R}^{d}\right) \longrightarrow L_{\gamma}^{p}\left(\mathbb{R}^{d}\right)$.

Item (1.) follows from Lemma B.1, and the result of item (2) from Lemma B.4. Then, the span of the kernel and cokernel of $\Delta^{m}(\mathrm{Id}-\Delta)^{l}$ can be determined using Lemma B.3 and duality.

We start by showing the operator $(\mathrm{Id}-\Delta)$ is an isomorphism in weighted spaces. As part of the proof we need the following Theorem by Kato, see Kat13.

Theorem (Kato, p.370). Let $T(\gamma)$ be a family of compact operators in a Banach space $X$ which are holomorphic for all $\gamma \in \mathbb{C}$. Call $\gamma$ a singular point if 1 is an eigenvalue of $T(\gamma)$. Then either all $\gamma \in D$ are singular points or there are only finitely many singular points in each compact subset of $D$.

With the notation $\langle\mathbf{x}\rangle=\left(1+|\mathbf{x}|^{2}\right)^{1 / 2}$, we are now ready to show that:

Lemma B.1. Given $s \in \mathbb{Z}, p \in(1, \infty), \gamma \in \mathbb{R}$, the operator $(\operatorname{Id}-\Delta): W_{\gamma}^{s, p}\left(\mathbb{R}^{d}\right) \rightarrow$ $W_{\gamma}^{s-2, p}\left(\mathbb{R}^{d}\right)$ is an isomorphism.

Proof. We show the result only for $s \geq 2$, since the results can be extended to all $s \in \mathbb{Z}$ by duality. We have the following commutative diagram

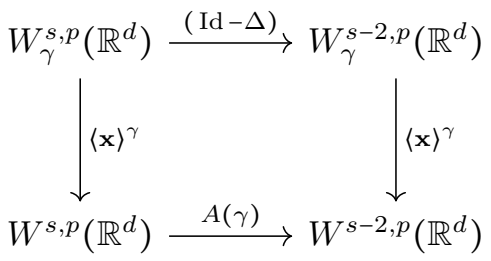

where $A(\gamma) u=(\operatorname{Id}-\Delta) u+T(\gamma) u$, and $T(\gamma) u=-\gamma(\gamma+2)|\mathbf{x}|^{2}\langle\mathbf{x}\rangle^{-4} u+\gamma\langle\mathbf{x}\rangle^{-2} u+$ $2 \gamma\langle\mathbf{x}\rangle^{-2} \mathbf{x} \cdot \nabla u$. The operator $T(\gamma)$ may be approximated using compactly supported functions. It then follows by the Rellich Kondrachov embedding theorem that $T(\gamma)$ is a compact perturbation of $(\mathrm{Id}-\Delta): W^{s, p}\left(\mathbb{R}^{d}\right) \rightarrow W^{s-2, p}\left(\mathbb{R}^{d}\right)$.

The results of the lemma follow if we can show that $\operatorname{Ker} A(\gamma)=\{0\}$ for all $\gamma \in \mathbb{R}$. Suppose for the moment that this is not true and that we can find a number $\gamma^{*} \in \mathbb{R}$ and a function $u \in W^{s, p}\left(\mathbb{R}^{d}\right)$ such that $A\left(\gamma^{*}\right) u=0$. Then, from the commutative diagram and the embedding $W_{\gamma^{*}}^{s, p}\left(\mathbb{R}^{d}\right) \subset W_{\gamma}^{s, p}\left(\mathbb{R}^{d}\right)$, with $\gamma<\gamma^{*}$, we obtain that $A(\gamma) u=0$ for all $\gamma<\gamma^{*}$.

On the other hand, the operator

$$
\begin{array}{clc}
W^{s, p}\left(\mathbb{R}^{d}\right) & \longrightarrow & W^{s-2, p}\left(\mathbb{R}^{d}\right) \\
u & \longmapsto & (\operatorname{Id}-\Delta)^{-1} T(\gamma) u,
\end{array}
$$

which is compact and analytic for all $\gamma \in \mathbb{C}$, has $\lambda=1$ as an eigenvalue if and only if $A(\gamma)$ has a non trivial kernel. We have therefore shown that $\lambda=1$ is an eigenvalue of $(\operatorname{Id}-\Delta)^{-1} T(\gamma)$ for all $\gamma<\gamma^{*}$. Now, Kato's Theorem implies that $\lambda=1$ is an eigenvalue of $(\operatorname{Id}-\Delta)^{-1} T(\gamma)$ for all $\gamma \in \mathbb{C}$. In particular, this result holds form $\gamma=0$, which is a contradiction as $(\mathrm{Id}-\Delta)$ is invertible. It therefore follows that $\operatorname{Ker} A(\gamma)=\{0\}$ for all $\gamma \in \mathbb{R}$ and that $(\operatorname{Id}-\Delta): W_{\gamma}^{s, p}\left(\mathbb{R}^{d}\right) \rightarrow W_{\gamma}^{s-2, p}\left(\mathbb{R}^{d}\right)$ is an isomorphism.

Before showing the Fredholm properties of $\Delta^{m}$ in Kondratiev spaces we need some results concerning homogenous polynomials. For more detailed proofs regarding the following results see [SW16. 
Homogenous Polynomials: Let $\mathbb{P}_{m}$ denote the space of complex valued homogenous polynomials in $\mathbb{R}^{d}$ of degree $m$, and let $\mathbb{H}_{m} \subset \mathbb{P}_{m}$ denote the subspace of harmonic polynomials. One can define a inner product in $\mathbb{P}_{m}$, via $\langle P, Q\rangle=P(D) \bar{Q}$, where the bar denotes complex conjugation and $P(D)$ is the differential operator by which $x_{i}$ is replaced with $\partial / \partial_{j}$. With this notion of an inner product it is possible to show that:

Lemma B.2. The space $\mathbb{P}_{m}$ is a direct sum of the form

$$
\mathbb{P}=\mathbb{H}_{m} \oplus|\mathbf{x}|^{2} \mathbb{H}_{m-2} \oplus|\mathbf{x}|^{4} \mathbb{H}_{m-4} \oplus \cdots \oplus|\mathbf{x}|^{2 \alpha} \mathbb{H}_{m-2 \alpha},
$$

where $\alpha$ is a positive integer such that $m=2 \alpha$ if $m$ is even, or $m+1=2 \alpha$ if $m$ is odd.

Since the dimension of $\mathbb{P}_{m}$ is given by $d_{m}=\left(\begin{array}{c}n+m-1 \\ m\end{array}\right)$, it follows from Lemma B.2 that the $\operatorname{dim}\left(\mathbb{H}_{m}\right)=\left(\begin{array}{c}n+m-1 \\ m\end{array}\right)-\left(\begin{array}{c}n+m-3 \\ m-2\end{array}\right)$.

The following notation,

$$
\mathcal{H}_{m, k}= \begin{cases}\mathbb{P}_{m} & \text { if } \quad m<2 k \\ \mathbb{H}_{m} \oplus|\mathbf{x}|^{2} \mathbb{H}_{m-2} \oplus \cdots \oplus|\mathbf{x}|^{2(k-1)} \mathbb{H}_{m-2(k-1)} & \text { if } \quad m \geq 2 k\end{cases}
$$

is used in the next lemma.

Lemma B.3. The operator $\Delta^{k}: \mathbb{P}_{m} \rightarrow \mathbb{P}_{m-2}$ is onto with kernel $\mathcal{H}_{m, k}$

Proof. The result is trivially true if $m \leq 2 k$. For the case $m>2 k$ suppose the result does not hold, so that there exist a $q(\mathbf{x}) \in \mathbb{P}_{m-2 k}$ such that $\left\langle\Delta^{k} p, q\right\rangle=0$ for all $p(\mathbf{x}) \in \mathbb{P}_{m}$. Take $p(\mathbf{x})=|\mathbf{x}|^{2 k} q(\mathbf{x})$ and notice that

$$
0=\overline{\left\langle\Delta^{k} p, q\right\rangle}=\left\langle q, \Delta^{k} p\right\rangle=q(D) \Delta^{k} \bar{p}=\Delta^{k} q(D) \bar{p}=\langle p, p\rangle .
$$

Since $\langle\cdot, \cdot\rangle$ is an inner product, it follows that $p(\mathbf{x}) \equiv 0$ and as a consequence the map $\Delta^{k}: \mathbb{P}_{m} \rightarrow \mathbb{P}_{m-2 k}$ is onto.

Now, let $r(\mathbf{x})=|\mathbf{x}|^{2 k} q(\mathbf{x})$ for some $q(\mathbf{x}) \in \mathbb{P}_{m-2 k}$, and take $p(\mathbf{x}) \in \mathbb{P}_{m}$ such that $\langle r, p\rangle=0$. This last equality holds if and only if

$$
\langle r, p\rangle=r(D) \bar{p}=q(D) \Delta^{k} \bar{p}=\left\langle q, \Delta^{k} p\right\rangle=0 .
$$

That is, if and only if $p(\mathbf{x}) \in \operatorname{Ker} \Delta^{k}$. Therefore, $\mathbb{P}_{m}=\operatorname{Ker}\left(\Delta^{k}\right) \oplus|\mathbf{x}|^{2 k} \mathbb{P}_{m-2 k}$, and by Lemma B.2 we must have

$$
\begin{aligned}
\operatorname{Ker}\left(\Delta^{k}\right) & =\mathbb{H}_{m} \oplus|\mathbf{x}|^{2} \mathbb{H}_{m-2} \oplus \cdots \oplus|\mathbf{x}|^{2(k-1)} \mathbb{H}_{m-2(k-1)} \\
& =\mathcal{H}_{m, k} .
\end{aligned}
$$

We are now ready to state the Fredholm properties of $\Delta^{m}: M_{\gamma-2 m}^{2 m, p}\left(\mathbb{R}^{d}\right) \rightarrow$ $L_{\gamma}^{p}\left(\mathbb{R}^{d}\right)$.

Lemma B.4. Given $\gamma \in \mathbb{R}, \alpha, \beta \in \mathbb{N} \cup\{0\}, m, n \in \mathbb{Z}^{+}$, and $p \in(1, \infty)$ the operator

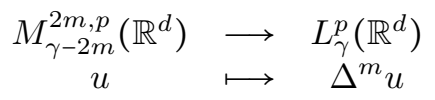

is

- Fredholm for $\alpha+n / p<\gamma<-\beta-n / p+2 m$ with kernel and cokernel given by

$$
\text { Ker }=\bigcup_{j=0}^{\beta} \mathcal{H}_{j, k} \quad \text { Coker }=\bigcup_{j=0}^{\alpha} \mathcal{H}_{j, k}
$$


- and it is not Fredholm for values of $\gamma \in\{j+n / p: j \in \mathbb{Z}\}$.

Proof. From the results in [McO79], it is straight forward to see that the operator is Fredholm. The span of the kernel and cokernel follows from Lemma B.3, while the range of values for $\gamma$ can be found by determining when the subspace $\mathbb{P}_{j}$ is contained in $\in L_{\gamma-2 m}^{p}\left(\mathbb{R}^{d}\right)$, or in $L_{-\gamma}^{p}\left(\mathbb{R}^{d}\right)$.

B.3. Fredholm properties of radial derivatives. In what follows, $\gamma \in \mathbb{R}$ and $p, q \in(1, \infty)$ are conjugate exponents. We also use the notation $\langle r\rangle=\left(1+r^{2}\right)^{1 / 2}$.

Lemma B.5. Let $\gamma \in \mathbb{R}$, and $p \in(1, \infty)$. Then the operator

$$
\begin{array}{clc}
W_{r, \gamma}^{1, p}\left(\mathbb{R}^{2}\right) & \longrightarrow & L_{r, \gamma}^{p}\left(\mathbb{R}^{2}\right) \\
u & \longmapsto & \partial_{r} u+\frac{1}{r} u-u
\end{array}
$$

is an invertible operator.

Proof. We show that the inverse operator

$$
\begin{array}{ccc}
L_{r, \gamma}^{p}\left(\mathbb{R}^{2}\right) & \longrightarrow & W_{r, \gamma}^{1, p}\left(\mathbb{R}^{2}\right) \\
f(r) & \longmapsto u(r)=\frac{1}{r} \int_{\infty}^{r} \mathrm{e}^{-(r-s)} f(s) s d s
\end{array}
$$

is a bounded operator using the following inequality

$$
\|u\|_{L_{\gamma}^{p}\left(\mathbb{R}^{2}\right)} \leq\|u\|_{L_{\gamma}^{p}\left(B_{1}\right)}+\|u\|_{L_{\gamma}^{p}\left(\mathbb{R}^{2} \backslash B_{1}\right)},
$$

where $B_{1}$ is the unit ball in $\mathbb{R}^{2}$. First, given $f \in C_{0}^{\infty}$ a simple calculation shows that $u(r)$ has the same type of singularity as $r f(r)$ near the origin. Since $C_{0}^{\infty}(\mathbb{R})$ is dense in $L_{\gamma}^{p}\left(\mathbb{R}^{2}\right)$, the result also holds for any $f \in L_{\gamma}^{p}\left(\mathbb{R}^{2}\right)$ and consequently,

$$
\|u\|_{L_{\gamma}^{p}\left(B_{1}\right)} \leq C\|f\|_{L_{\gamma}^{p}\left(\mathbb{R}^{2}\right)},
$$

as well as

$$
\|u(r) / r\|_{L_{\gamma}^{p}\left(B_{1}\right)} \leq C\|f\|_{L_{\gamma}^{p}\left(\mathbb{R}^{2}\right)} .
$$

On the other hand, because $\langle r\rangle^{\eta}\langle s\rangle^{-\eta} \leq\langle r-s\rangle^{|\eta|}$ holds for any $\eta \in \mathbb{R}$ we obtain

$$
\begin{aligned}
\|u\|_{L_{\gamma}^{p}\left(\mathbb{R}^{2} \backslash B_{1}\right)}^{p} & =\int_{1}^{\infty}\left|\frac{1}{r} \int_{\infty}^{r} \mathrm{e}^{(r-s)} f(s) s d s\right|^{p}\langle r\rangle^{\gamma p} r d r \\
& \leq \int_{1}^{\infty}\left|\int_{\infty}^{r} \mathrm{e}^{(r-s)}\langle r-s\rangle^{|\gamma-1+1 / p|} f(s)\langle s\rangle^{\gamma+1 / p} d s\right|^{p} d r
\end{aligned}
$$

from which we deduce using Young's inequality that $\|u\|_{L_{\gamma}^{p}\left(\mathbb{R}^{2} \backslash B_{1}\right)} \leq C(\gamma)\|f\|_{L_{\gamma}^{p}\left(\mathbb{R}^{2}\right)}$. It is then straightforward to see that $\left\|\partial_{r} u\right\|_{L_{\gamma}^{p}\left(\mathbb{R}^{2}\right)} \leq\|f\|_{L_{\gamma}^{p}\left(\mathbb{R}^{2}\right)}$, since $\partial_{r} u=u-\frac{1}{r} u-f$.

The next propositions states the Fredholm properties for $\partial_{r}$ and its adjoint $\partial_{r}+$ $1 / r$.

Proposition B.6. Given $p \in(1, \infty)$ the operator $\partial_{r}: M_{r, \gamma-1}^{k, p}\left(\mathbb{R}^{2}\right) \rightarrow L_{r, \gamma}^{p}\left(\mathbb{R}^{2}\right)$ is a Fredholm operator and

- for $\gamma>1-2 / p$ it is invertible, whereas

- for $\gamma<1-2 / p$ it is surjective with $\operatorname{Ker}=\{1\}$.

On the other hand, the operator does not have closed range for $\gamma=1-2 / p$.

Similarly,

Proposition B.7. Given $p \in(1, \infty)$, the operator $\partial_{r}+\frac{1}{r}: M_{r, \gamma-1}^{k, p}\left(\mathbb{R}^{2}\right) \rightarrow L_{r, \gamma}^{p}\left(\mathbb{R}^{2}\right)$ is a Fredholm operator and 
- for $\gamma>2-2 / p$ it is injective with Coker $=\{1\}$, whereas

- for $\gamma<2-2 / p$ it is invertible.

On the other hand, the operator does not have closed range for $\gamma=2-2 / p$.

The proof of the above propositions follows from the next lemmas and duality.

Lemma B.8. Given $\gamma>1-2 / p$ and $p \in(1, \infty)$, the operator $\partial_{r}: M_{r, \gamma-1}^{1, p}\left(\mathbb{R}^{2}\right) \rightarrow$ $L_{r, \gamma}^{p}\left(\mathbb{R}^{2}\right)$ is invertible.

Proof. We define the inverse operator

$$
\begin{aligned}
& \partial_{r}^{-1}: L_{r, \gamma}^{p}\left(\mathbb{R}^{2}\right) \quad \rightarrow \quad M_{r, \gamma-1}^{1, p}\left(\mathbb{R}^{2}\right) \\
& f(r) \quad \longmapsto u(r)=\int_{\infty}^{r} f(s) d s
\end{aligned}
$$

and show, using the following inequality

$$
\|u\|_{L_{\gamma-1}^{p}\left(\mathbb{R}^{2}\right)} \leq\|u\|_{L_{\gamma-1}^{p}\left(B_{1}\right)}+\|u\|_{L_{\gamma-1}^{p}\left(\mathbb{R}^{2} \backslash B_{1}\right)},
$$

where $B_{1}$ is the unit ball in $\mathbb{R}^{2}$, that $\|u\|_{L_{\gamma-1}^{p}\left(\mathbb{R}^{2}\right)}<\|f\|_{L_{\gamma}^{p}\left(\mathbb{R}^{2}\right)}$.

First, the result $\|u\|_{L_{\gamma-1}^{p}\left(B_{1}\right)} \leq\|f\|_{L_{\gamma}^{p}\left(B_{1}\right)}$ is a consequence of the Sobolev embeddings and the fact that $\langle r\rangle$ is a bounded function on $B_{1}$.

To show that $\|u\|_{L_{\gamma-1}^{p}\left(\mathbb{R}^{2} \backslash B_{1}\right)} \leq\|f\|_{L_{\gamma-1}^{p}\left(\mathbb{R}^{2} \backslash B_{1}\right)}$ consider the following scaling variables

$$
\tau=\ln (r), \quad r \in[1, \infty), \quad w(\tau)=u\left(\mathrm{e}^{\tau}\right) \mathrm{e}^{\sigma \tau}, \quad g(\tau)=f\left(\mathrm{e}^{\tau}\right) \mathrm{e}^{(\sigma+1) \tau}
$$

and notice that $w(\tau)$ satisfies $\partial_{\tau}\left(w \cdot \mathrm{e}^{-\sigma \tau}\right)=\mathrm{e}^{-\sigma \tau} g(\tau)$, hence $w(\tau)=\int_{\infty}^{\tau} \mathrm{e}^{\sigma(\tau-s)} g(s) d s$. Then, letting $\sigma=\gamma-1+2 / p>0$ and using Young's inequality we arrive at

$$
\|u\|_{L_{\gamma-1}^{p}\left(\mathbb{R}^{2} \backslash B_{1}\right)}=\|w\|_{L^{p}[1, \infty)} \leq \frac{1}{\sigma}\|g\|_{L^{p}[1, \infty)} \leq C\|f\|_{L_{\gamma}^{p}\left(\mathbb{R}^{2} \backslash B_{1}\right)} .
$$

Lemma B.9. Given $\gamma>2-2 / p$ and $p \in(1, \infty)$, the operator $\partial_{r}+\frac{1}{r}: M_{r, \gamma-1}^{1, p}\left(\mathbb{R}^{2}\right) \rightarrow$ $L_{r, \gamma}^{p}\left(\mathbb{R}^{2}\right)$ is Fredholm index $i=-1$ with Coker $=\{1\}$.

Proof. Since for $\gamma>2-2 / p$ the function 1 is in the dual of $L_{r, \gamma}^{p}\left(\mathbb{R}^{2}\right)$ and it defines a bounded linear functional. Therefore, the space

$$
L_{r, \gamma, \perp}^{p}=\left\{u \in L_{r, \gamma}^{p}\left(\mathbb{R}^{2}\right) \mid \int_{0}^{\infty} u \cdot r d r=0\right\}
$$

is a closed subspace. Moreover, given $u \in C_{0}^{\infty} \subset M_{\gamma-1}^{1, p}$ integration by parts shows that $\partial_{r} u+\frac{1}{r} u \in L_{r, \gamma, \perp}^{p}$. Then, because $\gamma>2-2 / p$ and $C_{0}^{\infty}$ is dense in $M_{\gamma-1}^{1, p}$ the same is true for any $u \in M_{r, \gamma-1}^{1, p}$. The results of the lemma then follow if one shows that the inverse operator

$$
\begin{aligned}
L_{r, \gamma, \perp}^{p} & \rightarrow \quad M_{r, \gamma}^{1, p}\left(\mathbb{R}^{2}\right) \\
f(r) & \longmapsto u(r)=\frac{1}{r} \int_{\infty}^{r} f(s) \cdot s d s
\end{aligned}
$$

is bounded. The proof of this last statement follows a similar arguments as in Lemma B.5 and Lemma B.8, and we therefore omit it. 
To complete the proof of Propositions B.6 and B.7 we need to define the extended operators $\partial_{r}: L_{r, \gamma-1}^{p}\left(\mathbb{R}^{2}\right) \rightarrow M_{\gamma}^{-1, p}\left(\mathbb{R}^{2}\right)$ and $\partial_{r}+\frac{1}{r}: L_{r, \gamma-1}^{p}\left(\mathbb{R}^{2}\right) \rightarrow M_{r, \gamma}^{-1, p}\left(\mathbb{R}^{2}\right)$ defined via

$$
\partial_{r} u(v)=\left\langle\left\langle u,\left(\partial_{r}\right)^{*} v\right\rangle\right\rangle=\left\langle\left\langle u,\left(\partial_{r}+1 / r\right) v\right\rangle, \quad \forall u \in L_{r, \gamma-1}^{p}\left(\mathbb{R}^{2}\right), \forall v \in M_{r,-\gamma}^{1, q}\left(\mathbb{R}^{2}\right)\right.
$$

where the double brackets $\langle\langle u, v\rangle$ denote the paring between an element $v \in X$ and a linear functional $u \in X^{*}$. Notice as well that the definition for these operators is a natural extension of $\partial_{r}: M_{r, \gamma-1}^{1, p}\left(\mathbb{R}^{2}\right) \rightarrow L_{r, \gamma}^{p}\left(\mathbb{R}^{2}\right)$, and $\partial_{r}+\frac{1}{r}: M_{r, \gamma-1}^{1, p}\left(\mathbb{R}^{2}\right) \rightarrow$ $L_{r, \gamma}^{p}\left(\mathbb{R}^{2}\right)$ since by duality

$$
\partial_{r} u(v)=\left\langle\left\langle u,\left(\partial_{r}\right)^{*} v\right\rangle\right\rangle=\left\langle\left\langle u,\left(\partial_{r}+1 / r\right) v\right\rangle, \quad \forall u \in M_{r, \gamma-1}^{1, p}\left(\mathbb{R}^{2}\right), \forall v \in M_{r, 1-\gamma}^{1, q}\left(\mathbb{R}^{2}\right)\right.
$$

Lemma B.10. Let $p \in(1, \infty)$, then the operator $\partial_{r}: L_{r, \gamma-1}^{p}\left(\mathbb{R}^{2}\right) \rightarrow M_{r, \gamma}^{-1, p}\left(\mathbb{R}^{2}\right)$ is

- injective for $\gamma>1-2 / p$.

- Fredholm with Ker $=\{1\}$ and index $i=1$ for $\gamma<1-2 / p$.

Proof. To show it is injective for $\gamma>1-2 / p$, suppose there is a $u \in L_{r, \gamma-1}^{p}\left(\mathbb{R}^{2}\right)$ such that $\partial_{r} u=0$. Then using a sequence $\left\{u_{n}\right\} \in C_{0}^{\infty}\left(\mathbb{R}^{2}\right)$ such that $u_{n} \rightarrow u$ in $L_{r, \gamma-1}^{p}\left(\mathbb{R}^{2}\right)$ we find that for all $v \in M_{r,-\gamma}^{1, q}\left(\mathbb{R}^{2}\right)$

$$
0=\partial_{r} u=\left\langle\left\langle u,\left(\partial_{r}\right)^{*} v\right\rangle\right\rangle=\lim _{n \rightarrow \infty}\left\langle\left\langle\partial_{r} u_{n}, v\right\rangle\right\rangle .
$$

It follows that $\partial_{r} u_{n} \rightarrow 0$ in $L_{r, \gamma}^{p}$. Since $\gamma>1-2 / p$ we must have $u=0$.

The result for $\gamma<1-2 / p$ follows from the definition $\partial_{r} u(v)=\left\langle\left\langle u,\left(\partial_{r}+1 / r\right) v\right\rangle\right.$, and Lemma B.9.

Similar arguments as in the above lemma show that

Lemma B.11. Given $p \in(1, \infty)$, the operator $\partial_{r}+\frac{1}{r}: L_{r, \gamma-1}^{p}\left(\mathbb{R}^{2}\right) \rightarrow M_{r, \gamma}^{-1, p}\left(\mathbb{R}^{2}\right)$ is

- injective for $\gamma<2-2 / p$.

- Fredholm with Coker $=\{1\}$ and index $i=-1$ for $\gamma>2-2 / p$.

Lemma B.12. Given $\gamma=1-2 / p$, the operator $\partial_{r}: M_{r, \gamma-1}^{1, p}\left(\mathbb{R}^{2}\right) \rightarrow L_{r, \gamma}^{p}\left(\mathbb{R}^{2}\right)$ does not have a closed range.

Proof. Let $\psi(r) \in C_{0}^{\infty}\left(\mathbb{R}^{2}\right)$ be a radial function with $\operatorname{supp} \psi(r) \in B_{1}$ and $\psi(r)=1$ for $r<1 / 2$. Define $u_{n}(r)=\psi(r / n) /\|\psi(r / n)\|_{L_{r, \gamma-1}^{p}}$ for $n \in \mathbb{N}$ and notice that $\partial_{r} u_{n} \rightarrow 0$ in $L_{r, \gamma}^{p}\left(\mathbb{R}^{2}\right)$ yet for $\gamma=1-2 / p$ the sequence $\left\{u_{n}\right\}$ does not converge in $L_{r, \gamma-1}^{p}\left(\mathbb{R}^{2}\right)$. It follows then that $\partial_{r}: M_{r, \gamma-1}^{1, p}\left(\mathbb{R}^{2}\right) \rightarrow L_{r, \gamma}^{p}\left(\mathbb{R}^{2}\right)$ does not have closed range.

Lemma B.13. Given $\gamma=1-2 / p$, the operator $\partial_{r}+\frac{1}{r}: M_{r, \gamma-1}^{1, p}\left(\mathbb{R}^{2}\right) \rightarrow L_{r, \gamma}^{p}\left(\mathbb{R}^{2}\right)$ does not have a closed range.

Proof. The proof is similar to that of Lemma B.12 only we use the following sequence instead: Let $\psi(r) \in C_{0}^{\infty}\left(\mathbb{R}^{2}\right)$ be a radial function with $\operatorname{supp} \psi(r) \in B_{2}$ and $\psi(r)=1 / r$ for $1<r<3 / 2$, and $\psi(r)=0$ for $r<1 / 2$. Then define $u_{n}(r)=$ $\psi(r / n) /\|\psi(r / n)\|_{L_{r, \gamma-1}^{p}\left(\mathbb{R}^{2}\right)}$. 


\section{REFERENCES}

[ADGK $\left.{ }^{+} 08\right]$ Alex Arenas, Albert Díaz-Guilera, Jurgen Kurths, Yamir Moreno, and Changsong Zhou, Synchronization in complex networks, Physics reports 469 (2008), no. 3, 93153.

[AF03] Robert A Adams and John JF Fournier, Sobolev spaces, vol. 140, Academic press, 2003.

[AK02] Igor S Aranson and Lorenz Kramer, The world of the complex Ginzburg-Landau equation, Reviews of Modern Physics 74 (2002), no. 1, 99.

[AS92] Milton Abramowitz and Irene A. Stegun (eds.), Handbook of mathematical functions with formulas, graphs, and mathematical tables, Dover Publications Inc., New York, 1992, Reprint of the 1972 edition.

[AT06] Igor S Aranson and Lev S Tsimring, Patterns and collective behavior in granular media: Theoretical concepts, Reviews of modern physics 78 (2006), no. 2, 641.

[Boy99] John P. Boyd, The Devil's invention: Asymptotic, superasymptotic and hyperasymptotic series, Acta Appl. Math. 56 (1999), no. 1, 1-98.

[CBC81] Yvonne Choquet-Bruhat and Demetrios Christodoulou, Elliptic systems in $H_{s, \delta}$ spaces on manifolds which are Euclidean at infinity, Acta Mathematica 146 (1981), no. $1,129-150$.

[CH93] M. C. Cross and P. C. Hohenberg, Pattern formation outside of equilibrium, Rev. Mod. Phys. 65 (1993), 851-1112.

[CH09] Nicholas T. Carnevale and Michael L. Hines, The NEURON book, 1st ed., Cambridge University Press, New York, NY, USA, 2009.

[CM02] S.M. Cox and P.C. Matthews, Exponential time differencing for stiff systems, J. Comput. Phys. 176 (2002), no. 2, 430-455.

[Coo05] Stephen Coombes, Waves, bumps, and patterns in neural field theories, Biological cybernetics 93 (2005), no. 2, 91-108.

[DSSS05] Arjen Doelman, Björn Sandstede, Arnd Scheel, and Guido Schneider, The dynamics of modulated wave trains, CWI. Modelling, Analysis and Simulation [MAS], 2005.

[ER99] Jens Eggers and Hermann Riecke, Continuum description of vibrated sand, Phys. Rev. E 59 (1999), 4476-4483.

[Erm98] Bard Ermentrout, Neural networks as spatio-temporal pattern-forming systems, Reports on progress in physics 61 (1998), no. 4, 353.

[FB04] Stefanos E Folias and Paul C Bressloff, Breathing pulses in an excitatory neural network, SIAM Journal on Applied Dynamical Systems 3 (2004), no. 3, 378-407.

[Fis37] R. A. Fisher, The wave of advance of advantageous genes, Annals of Eugenics 7 (1937), no. 4, 355-369.

[Fit61] Richard FitzHugh, Impulses and physiological states in theoretical models of nerve membrane, Biophysical journal 1 (1961), no. 6, 445-466.

[GJP $\left.{ }^{+} 95\right]$ Richard A Gray, Jose Jalife, Alexandre V Panfilov, William T Baxter, Candido Cabo, Jorge M Davidenko, Arkady M Pertsov, Paulien Hogeweg, and Arthur T Winfree, Mechanisms of cardiac fibrillation, Science 270 (1995), no. 5239, 1222-1226.

[GKS00] M. Golubitsky, E. Knobloch, and I. Stewart, Target patterns and spirals in planar reaction-diffusion systems, Journal of Nonlinear Science 10 (2000), no. 3, 333-354.

[GSS88] Martin Golubitsky, Ian Stewart, and David Schaeffer, Singularities and groups in bifurcation theory : Volume II, Springer, New York, NY, 1988.

[Hag81] Patrick S Hagan, Target patterns in reaction-diffusion systems, Advances in Applied Mathematics 2 (1981), no. 4, $400-416$.

$\left[\mathrm{HTY}^{+}\right.$04] Xiaoying Huang, William C Troy, Qian Yang, Hongtao Ma, Carlo R Laing, Steven J Schiff, and Jian-Young $\mathrm{Wu}$, Spiral waves in disinhibited mammalian neocortex, The Journal of Neuroscience 24 (2004), no. 44, 9897-9902.

[Jar15] Gabriela Jaramillo, Inhomogeneities in 3 dimensional oscillatory media, Netw. Heterog. Media 10 (2015), no. 2, 387-399.

[Jar18] _ Target patterns, GitHub repository (2018), Available at https://doi.org/ 10.5281/zenodo.1239861, version 1.0.0.

[JS16] Gabriela Jaramillo and Arnd Scheel, Pacemakers in large arrays of oscillators with nonlocal coupling, Journal of Differential Equations 260 (2016), no. 3, 2060-2090. 
[JSW17] Gabriela Jaramillo, Arnd Scheel, and Qiliang Wu, The effect of impurities on striped phases, Proc. Roy. Soc. Edinb. (2017), to appear.

[Kat13] Tosio Kato, Perturbation theory for linear operators, vol. 132, Springer Science \& Business Media, 2013.

[KB02] Yoshiki Kuramoto and Dorjsuren Battogtokh, Coexistence of coherence and incoherence in nonlocally coupled phase oscillators., Nonlin. Phenom. Comp. Sys. 5 (2002), no. 4, 380-385.

[KB10a] Zachary P Kilpatrick and Paul C Bressloff, Effects of synaptic depression and adaptation on spatiotemporal dynamics of an excitatory neuronal network, Physica D: Nonlinear Phenomena 239 (2010), no. 9, 547-560.

[KB10b] _ Spatially structured oscillations in a two-dimensional excitatory neuronal network with synaptic depression, Journal of computational neuroscience 28 (2010), no. 2, 193-209.

[KH81] N Kopell and L. N Howard, Target pattern and spiral solutions to reaction-diffusion equations with more than one space dimension, Advances in Applied Mathematics 2 (1981), no. 4, 417-449.

[KM04] Hiroshi Kori and Alexander S Mikhailov, Entrainment of randomly coupled oscillator networks by a pacemaker, Physical review letters 93 (2004), no. 25, 254101.

[KPP37] Andrei Nikolaevitch Kolmogorov, Ivan G Petrovskii, and Nikolaï S Piskunov, A study of the equation of diffusion with increase in the quantity of matter, and its application to a biological problem, Bjul. Moskovskogo Gos. Univ (Bulletin Moscow State University) 1 (1937), no. 7, 1-26.

[KS07] Richard Kollár and Arnd Scheel, Coherent structures generated by inhomogeneities in oscillatory media, SIAM Journal on Applied Dynamical Systems 6 (2007), no. 1, 236-262.

[KT76] Yoshiki Kuramoto and Toshio Tsuzuki, Persistent propagation of concentration waves in dissipative media far from thermal equilibrium, Progress of Theoretical Physics 55 (1976), no. 2, 356-369.

[KT05] A. Kassam and L. Trefethen, Fourth-Order time-stepping for stiff PDEs, SIAM Journal on Scientific Computing 26 (2005), no. 4, 1214-1233.

[Kur75] Yoshiki Kuramoto, Self-entrainment of a population of coupled non-linear oscillators, International Symposium on Mathematical Problems in Theoretical Physics: January 23-29, 1975, Kyoto University, Kyoto/Japan (Huzihiro Araki, ed.), Springer Berlin Heidelberg, Berlin, Heidelberg, 1975, pp. 420-422.

[Kur84] Chemical Oscillations, Waves, and Turbulence, Springer Series in Synergetics, vol. 19, Springer Berlin Heidelberg, Berlin, Heidelberg, 1984.

[LT03] Carlo R Laing and William C Troy, PDE methods for nonlocal models, SIAM Journal on Applied Dynamical Systems 2 (2003), no. 3, 487-516.

[McO79] Robert C McOwen, The behavior of the Laplacian on weighted Sobolev spaces, Communications on Pure and Applied Mathematics 32 (1979), no. 6, 783-795.

[Mer92] Ehud Meron, Pattern formation in excitable media, Physics Reports 218 (1992), no. $1,1-66$.

[MM95] Osamu Moriyama and Mitsugu Matsushita, Simple model for target patterns and spiral waves, Journal of the Physical Society of Japan 64 (1995), no. 4, 1081-1084.

[Nag91] Hiroyuki Nagashima, Target patterns and pacemakersin a reaction-diffusion system, Journal of the Physical Society of Japan 60 (1991), no. 9, 2797-2799.

[NAY62] Jinichi Nagumo, Suguru Arimoto, and Shuji Yoshizawa, An active pulse transmission line simulating nerve axon, Proceedings of the IEEE 50 (1962), no. 10, 2061-2070.

[NW69] Alan C. Newell and J. A. Whitehead, Finite bandwidth, finite amplitude convection, J. Fluid Mech. 38 (1969), no. 2, 279-303.

[PE01] David J Pinto and G Bard Ermentrout, Spatially structured activity in synaptically coupled neuronal networks: I. Traveling fronts and pulses, SIAM journal on Applied Mathematics 62 (2001), no. 1, 206-225.

[PV87] A Pagola and C Vidal, Wave profile and speed near the core of a target pattern in the Belousov-Zhabotinsky reaction, Journal of Physical Chemistry 91 (1987), no. 3, 501-503.

[RMO06] Filippo Radicchi and Hildegard Meyer-Ortmanns, Entrainment of coupled oscillators on regular networks by pacemakers, Physical Review E 73 (2006), no. 3, 036218. 
[ROH05] Juan G Restrepo, Edward Ott, and Brian R Hunt, Onset of synchronization in large networks of coupled oscillators, Physical Review E 71 (2005), no. 3, 036151.

[Rou04] Violaine Roussier, Stability of radially symmetric travelling waves in reactiondiffusion equations, Annales de l'Institut Henri Poincare (C) Non Linear Analysis 21 (2004), no. 3, $341-379$.

[Seg69] Lee A. Segel, Distant side-walls cause slow amplitude modulation of cellular convection, J. Fluid Mech. 38 (1969), no. 1, 203-224.

[Sim76] Barry Simon, The bound state of weakly coupled Schrödinger operators in one and two dimensions, Annals of Physics 97 (1976), no. 2, 279-288.

[SK04] Shin-ichiro Shima and Yoshiki Kuramoto, Rotating spiral waves with phaserandomized core in nonlocally coupled oscillators, Physical Review E 69 (2004), no. 3, 036213.

[SL12] Michael A. Schwemmer and Timothy J. Lewis, The Theory of Weakly Coupled Oscillators, Phase Response Curves in Neuroscience: Theory, Experiment, and Analysis (Nathan W. Schultheiss, Astrid A. Prinz, and Robert J. Butera, eds.), Springer New York, New York, NY, 2012, pp. 3-31.

[SM06] Michael Stich and Alexander S Mikhailov, Target patterns in two-dimensional heterogeneous oscillatory reaction-diffusion systems, Physica D: Nonlinear Phenomena 215 (2006), no. 1, 38-45.

[SW16] Elias M Stein and Guido Weiss, Introduction to Fourier analysis on Euclidean spaces, vol. 32, Princeton university press, 2016.

[TF80a] John J Tyson and Paul C Fife, Target patterns in a realistic model of the BelousovZhabotinskii reaction, The Journal of Chemical Physics 73 (1980), no. 5, 2224-2237.

[TF80b] John J. Tyson and Paul C. Fife, Target patterns in a realistic model of the BelousovZhabotinskii reaction, The Journal of Chemical Physics 73 (1980), no. 5, 2224-2237.

[UMS98] Paul B. Umbanhowar, Francisco Melo, and Harry L. Swinney, Periodic, aperiodic, and transient patterns in vibrated granular layers, Physica A: Statistical Mechanics and its Applications 249 (1998), no. 1-4, 1-9.

[VO98] Shankar C. Venkataramani and Edward Ott, Spatiotemporal bifurcation phenomena with temporal period doubling: Patterns in vibrated sand, Phys. Rev. Lett. 80 (1998), 3495-3498.

[VO01] _ Pattern selection in extended periodically forced systems: A continuum coupled map approach, Phys. Rev. E 63 (2001), 046202.

[Win73] Arthur T. Winfree, Scroll-Shaped waves of chemical activity in three dimensions, Science 181 (1973), no. 4103, 937-939.

[WW96] E. T. Whittaker and G. N. Watson, A course of modern analysis, Cambridge Mathematical Library, Cambridge University Press, Cambridge, 1996, Reprint of the fourth (1927) edition.

[YHLZ13] Nan Yao, Zi-Gang Huang, Ying-Cheng Lai, and Zhi-Gang Zheng, Robustness of chimera states in complex dynamical systems, Scientific reports 3 (2013), 3522.

Department of Mathematics, University of Arizona, Tucson, AZ 85721

E-MAIL: gjaramillo@math.arizona.edu

Department of Mathematics, University of Arizona, Tucson, AZ 85721

E-MAIL: shankar@math.arizona.edu 